\title{
Characterization of the energy level-structure of a trapped dipolar Bose gas via mean-field parametric resonances
}

\author{
Asaad R. Sakhel ${ }^{1}$ and Roger R. Sakhel ${ }^{2}$ \\ ${ }^{1}$ Department of Physics, Faculty of Science, Balqa Applied University, Salt 19117, Jordan \\ ${ }^{2}$ Department of Physics, Faculty of Science, Isra University, Amman 11622, Jordan
}

(Dated: January 26, 2021)

\begin{abstract}
We report parametric resonances (PRs) in the mean-field dynamics of a one-dimensional dipolar Bose-Einstein condensate (DBEC) in widely varying trapping geometries. The chief goal is to characterize the energy levels of this system by analytical methods and the significance of this study arises from the commonly known fact that in the presence of interactions the energy levels of a trapped BEC are hard to calculate analytically. The latter characterization is achieved by a matching of the PR energies to energy levels of the confining trap using perturbative methods. Further, this work reveals the role of the interplay between dipole-dipole interactions (DDI) and trapping geometry in defining the energies and amplitudes of the PRs. The PRs are induced by a negative Gaussian potential whose depth oscillates with time. Moreover, the DDI play a role in this induction. The dynamics of this system is modeled by the time-dependent Gross- Pitaevskii equation (TDGPE) that is numerically solved by the Crank-Nicolson method. The PRs are discussed basing on analytical methods: first, it is shown that it is possible to reproduce PRs by the Lagrangian variational method that are similar to the ones obtained from TDGPE. Second, the energies at which the PRs arise are closely matched with the energy levels of the corresponding trap calculated by time-independent perturbation theory. Third, the most probable transitions between the trap energy levels yielding PRs are determined by time-dependent perturbation theory. The most significant result of this work is that we have been able to characterize the above mentioned energy levels of a DBEC in a complex trapping potential.
\end{abstract}

Keywords: Dipolar Bose-Einstein condensates, variational methods, Crank-Nicolson method, parametric resonances

\section{INTRODUCTION}

The phenomenon of parametric resonances (PRs) is ubiquitious in nature and is a widely examined fundamental physical property. Today, PRs are one of the outstanding features observed in Bose-Einstein condensates (BECs) [1 18] where resonances are usually the result of modulating one of the system parameters such as the scattering length [1, 19 22]. They are also generated by external means such as a time-dependent trapping geometry [11], laser stirring [2], and laser-intensity modulation [1]. Moreover, PRs have been shown to occur in classical systems [23, 24] as well as quantum devices such as birefringent optical fibers [25], magnetometers [26], superconducting wave guides [27], and quantum dots [28, 29]. Their importance has also been demonstrated in transatlantic telecommunication fiber optics [30] in connection to a modulational instability.

In this work, we explore PRs in a dipolar BEC (DBEC) within a setting of large-sized traps that would allow an examination of the effects of long-range interactions. It is known that dipole-dipole interactions (DDI) are capable of shifting the frequency of PRs [1, 31, 32]. This shift is sensitive to the trapping geometry [31]. As a result, one concludes that the interplay between trapping geometry and DDI determines the energies at which PRs occur. These energies are equivalent to the energies of the trap levels; the trap in which the DBEC is confined. Within this context then, the chief goal is to characterize the energy-level structure (ELS) of a DBEC in a trap of large size by matching the PR energies to energy levels of this trap using perturbative methods. Herein, the importance of PRs is revealed in characterizing the shape of the BEC trap and its energy levels [1]. The significance of the present investigation arises from the fact that it facilitates an evaluation of the above-named structure in complex potentials. This is because it is known that, in the presence of interactions, it is rather hard to analytically calculate the energy levels of a trapped BEC. Moreover, via the present research, we hope to motivate future experiments that could determine the ELS of more complicated traps that may be engineered in the future. That said, the usefulness of the present examination is revealed from the latter statements. Another goal is to reveal the interplay between trapping geometry and DDI (cf. [20, 33 35]) in defining the frequencies at which PRs occur. Effects of trapping geometry have already been studied earlier such as the influence of the trap aspect ratio on the oscillation frequencies [36] and stability of a DBEC [37, 38], except that it hasn't been related to the structure of the trap energy levels.

At present, we consider a one-dimensional (1D) trapped DBEC that is driven by a negative Gaussian potential (NGP) whose depth is periodically modulated with time. The latter system is simulated numerically in different traps using the mean-field time-dependent Gross-Pitaveskii equation (TDGPE). The 1D DBEC is scanned over a long range of DDI strengths in an attempt to detect PRs and to study the above mentioned interplay. In passing, it should be noted that the 1D Bose 
gas is a general, important, and well known system that can refer to many physical systems, such as optical fibers [25, 39 41]. It is studied to reveal the physics in 1D which is strikingly different than in higher dimensions. The addition of an NGP is for the purpose of causing the particles to condense into lower energy levels, so to speak to "catch particles", and then throw (excite) them to higher energy levels by the oscillating NGP depth. The oscillatory NGP has been earlier shown to work like a modulated contact interaction [7] and could therefore be viewed analogous to it. It should also be noted that an NGP has been used to induce a BEC and to study its growth dynamics 42. With the above NGP, a laserlight source is modelled whose intensity oscillates with time [1, 43] and provides a softer stirring of the BEC in energy space than the stirring by time-dependent spatial modulations. This method has rarely been used or mentioned in the BEC literature, and here it is demonstrated that it leads to significant excitations.

The PRs are measured by a quantity that resembles the time average of the square of the kinetic energy called "signal energy" 44]. The signal energy is a term borrowed from engineering topics for the processing of an oscillating electrical signal. The signal energy has also been found very effective in revealing PRs in one of our earlier publications [2].

One motivation for the present examination arises from the work of Balik et al. [1] who applied a $\mathrm{CO}_{2-}$ laser generated optical-dipole trap to confine a sample of ${ }^{87} \mathrm{Rb}$ atoms. By a modulation of the laser intensity, the authors were able to excite PRs whose frequencies were found to shift by a change of the laser's modulation depth. In this regard, we make an analogy by explaining the frequency shift as resulting from a change in the depth of the effective time-averaged potential of the trapped DBEC. The latter depth is controlled by the DDI, and by scanning the DBEC over a broad range of the latter it has been possible to observe PRs at values of DDI corresponding to the resonance frequencies. This project has been heavily computational as it required a large number of runs to locate the DDI regimes where PRs occur.

The most significant result of this work is that we have been able to characterize the energy levels of a DBEC in a complex trapping potential, i.e., a trap to which an NGP is added. Other key results are detailed as follows.

(i) By a variation of the DDI strength we detect meanfield PRs in the dynamics of a DBEC induced by an NGP whose depth is periodically modulated with time. The PRs obtained are an inherent feature deeply embedded in the physics of the DBEC as they could also be generated by other tools such as the Lagrangian variational method (LVM) $7,45-$ 51].

(ii) The positions of the PRs are determined by the ELS of the trapping geometry. It is shown that the energies at which PRs occur can be characterized by second-order perturbation theory thus allowing us to determine the above mentioned structure.

(iii) It is shown that the depth of the time-averaged effective potential is reduced by an increase in the repulsive DDI strength as a result of which the PR frequencies are shifted to larger values in the case of a harmonic oscillator (HO)+NGP. However, in the case of a BOX+NGP, the PR frequencies drop instead with rising DDI strength. Thus the interplay between trapping geometry and DDI controls the values of the DDI strength at which PRs arise in the signal energy [44]. Moreover, the amplitude of the PRs decline with increasing DDI because the depth of the time-averaged mean-field effective potential is reduced with it.

(iv) It is found that the occurrence of a $\mathrm{PR}$ requires two conditions: (1) its energy should closely match one of the trap levels and (2) the transition probability to this level should be quite large.

The organization of this paper is as follows. In Sec. III the method is presented. In Sec. III the results are presented and discussed and in Sec. IV the origins of the PRs are explained. Finally in Sec. $\nabla$ we summarize and conclude. In Appendix $\mathrm{A}$ technical details for the simulations are outlined and in Appendix B a measurement unit is derived.

\section{METHOD}

In this section, only the rudiments of the method are outlined. The reader is therefore referred to Ref.[52] for more details on the method and the numerics involved.

\section{A. Basic Units}

In the present work, lengths and energies are in units of the trap $a_{h o}=\sqrt{\hbar /(m \bar{\omega})}$ and $\hbar \bar{\omega}$, respectively, where $\bar{\omega}=\left(\omega_{x} \omega_{y} \omega_{z}\right)^{1 / 3}$ is the geometric average of the trapping frequencies along the coordinate axes, and $m$ is the mass of the atom. It should be however noted, that the modes in the $y$ - and $z$-directions are frozen since the present system is described by the TDGPE that is reduced from $3 \mathrm{D}$ to $1 \mathrm{D}$ by integrating out the transverse directions. Time is unitless where $t=\bar{\omega} \tau, \tau$ being the time in seconds.

\section{B. Systems}

The systems considered are 1D strongly repulsive DBECs that are confined in a few different trapping geometries. The confining potentials are power-law traps that have the form 


$$
V_{t r}(x)=\frac{1}{2}\left|\frac{x}{L_{x}}\right|^{p},
$$

where $p$ is the trapping exponent, and $L_{x}$ a length scale that shapes $V_{t r}(x)$ so that there is some flatness in the neighborhood of $x=0$ when $p$ is much larger than 2 . $V_{t r}(x)$ is in units of $\hbar \bar{\omega}$ whereas $x$ and $L_{x}$ are in $a_{h o}$. It should be emphasized that power-law traps have been realized in the United Kingdom using spatial light modulation [53].

The DBECs are excited by an NGP whose depth oscillates with time. Experimentally, the NGP is generated by the application of a focusing red-laser beam [42, 5461]. The NGP has been considered in theoretical investigations [62 67] as well. Its importance has been demonstrated in an architecture of quantum computation [66], the transportation of BECs [59], and the extraction of atoms from a BEC [63, 67]. Solitons [68] in an NGP and properties of a BEC in a harmonic trap plus an eccentric NGP [65] have also been examined. The red-detuned laser beam interacts with the BEC in such a way so as to introduce an NGP into it by phase-imprinting [69]. Adding to this the requirement of an oscillating intensity, the NGP is modelled by

$$
V_{D T}(x, t)=[A+\delta A \cos (\Omega t)] \exp \left(-\beta x^{2}\right),
$$

where $\Omega=2 \pi f$ is the driving frequency, $A$ the principal depth, $\delta A$ the modulation amplitude, and as usual $1 / \sqrt{\beta}$ a measure of the NGP width. $A$ and $\delta A$ are in units of $\hbar \bar{\omega}, \beta$ in $a_{h o}^{-2}$, and $\Omega$ is in units of $\bar{\omega}$. As already noted in the introduction, the analog of the present excitation method is the periodic modulation of a scattering length such as $a(t)=a_{b g}+\delta a \sin (\omega t)$ [19 21], where $\delta a$ is the modulation amplitude, and $a_{b g}$ an unperturbed background. The analog of $a_{b g}$ is the $A$ and of $\delta a$ the $\delta A$. In one of our earlier publications [7], it has been verified that the effects on the BEC arising from the NGP with oscillating depth [Eq.(2)] are indeed similar to those from $a(t)$ above if $a_{b g}<0$.

The time-modulation of the NGP depth in (2), that is $\delta V_{D T}(x, t)=\delta A \cos (\Omega t) e^{-\beta x^{2}}$, generates an oscillating force along the length of the DBEC that is given by the potential gradient

$$
\delta F_{x}=-\frac{\partial \delta V_{D T}(x, t)}{\partial x}=2 \beta x \delta A \cos (\Omega t) e^{-\beta x^{2}} .
$$

This force transfers a momentum from the oscillating NGP to the BEC that is symmetric about $x=0$ and reads

$$
\Delta p_{x}=\int_{0}^{t} \delta F_{x} d t=2 \beta x e^{-\beta x^{2}} \frac{\delta A \sin (\Omega t)}{\Omega} .
$$

The latter is maximal at $x= \pm 1 / \sqrt{2 \beta}$ and minimal at $x=0$ and the edges of the trap.

\section{Dipolar Interactions and their Control}

A note on the manipulation of the DDI is in order here. Consider the DDI potential given by [52, 70]

$$
U_{d d}(R)=C_{d d} \frac{\left(1-3 \cos ^{2} \theta\right)}{|\mathbf{R}|^{3}}
$$

where $\mathbf{R}=\mathbf{r}-\mathbf{r}^{\prime}$ is the relative position vector of two dipoles at $\mathbf{r}$ and $\mathbf{r}^{\prime}, \theta$ the angle between $\mathbf{R}$ and the orientation of the dipoles, $C_{d d}=E^{2} \alpha^{2} /\left(\hbar \bar{\omega} \epsilon_{0}\right)$ [70, 71] (in trap units) with $E$ being an external electric field, $\epsilon_{0}$ the permittivity of free space, and $\alpha$ the static polarizability. Thus, the DDI can then be induced and tuned by an external field $E$ [72] to various orders of magnitude 73] and in polar molecules the dipole moment can be set up to $10^{4}$ times larger than in atomic systems. The DDI also occur naturally 74 78] if the atoms possess a magnetic dipole moment $\bar{\mu}$ in which case $C_{d d}=\mu_{0} \bar{\mu}^{2} /(4 \pi \hbar \bar{\omega})$ [52, 70, 76] (in trap units) with $\mu_{0}$ the permittivity of free space. The control of DDI has also been further explained in the article by Lahaye et al. [70]. Moreover, by using the linear Stark effect, it is possible to excite Rydbergdressed atoms to very high principle quantum numbers to achieve large dipole moments like $p \sim 1450 D$, where $D$ is the ratio between the dipolar and $s$-wave scattering length [79]. In this regard, we justify the use of large values of the DDI parameter $\mathcal{G}_{1 d d}$ in the present work. Before we continue, it should be emphasized that the DDI in the present work is repulsive.

\section{Mean-Field Gross-Pitaevskii Equation}

The trapped DBEC is described by the timedependent Gross-Pitaevskii equation (TDGPE). It is reduced from the $3 \mathrm{D}$ to the $1 \mathrm{D}$ form by integrating out the contributions in the transverse direction (see e.g. Refs. [52, 80]). We thus consider a cigar-shaped DBEC that is elongated along the $x$-axis with strong radial confinement in the transverse direction. The dynamics in the transverse direction is frozen in the radial ground state

$$
\phi(\rho)=\frac{1}{d_{\rho} \sqrt{\pi}} e^{-\rho^{2} /\left(2 d_{\rho}^{2}\right)},
$$

where $d_{\rho}$ is the width of the Gaussian. The reduced 1D TDGPE reads then as in Ref. 52]

$$
\begin{aligned}
& i \frac{\partial \psi(x, t)}{\partial t}=\left[-\frac{1}{2} \frac{\partial^{2}}{\partial x^{2}}+V_{t r}(x)+V_{D T}(x, t)+\right. \\
& \mathcal{G}_{1 D}|\psi(x, t)|^{2}+ \\
& \left.\frac{4 \pi}{3} \mathcal{G}_{1 d d} \int_{-\infty}^{+\infty} \frac{d k_{x}}{2 \pi} e^{-i k_{x} x}\left|\widetilde{\psi}\left(k_{x}, t\right)\right|^{2} j_{1 D}\left(\tau_{x}\right)\right] \psi(x, t),
\end{aligned}
$$


where $\widetilde{\psi}\left(k_{x}, t\right)$ is the Fourier transform of $\psi(x, t)$. The latter is the longitudinal wave function which is normalized to one vis-á-vis $\int_{-\infty}^{+\infty}|\psi(x, t)|^{2} d x=1$ with $\psi(x, t)$ in units of $a_{h o}^{-1 / 2} \cdot j_{1 D}\left(\tau_{x}\right)$ is a function given by

$$
\begin{gathered}
j_{1 D}\left(\tau_{x}\right)=\frac{\sqrt{2}}{2 \pi d_{\rho}} \int_{-\infty}^{+\infty} d \tau_{y} e^{-\tau_{y}^{2}} h_{2 D}(\tau), \\
\text { with } \tau_{y}=d_{\rho} k_{y} / \sqrt{2}, \tau=\sqrt{\tau_{x}^{2}+\tau_{y}^{2}} \text {, and } \\
h_{2 D}(\tau)=\frac{1}{\sqrt{2 \pi} d_{\rho}}\left[2-3 \sqrt{\pi} e^{\tau^{2}} \tau\{1-\operatorname{erf}(\tau)\}\right] .
\end{gathered}
$$

$\mathcal{G}_{1 D}$ and $\mathcal{G}_{1 d d}$ are respectively the $1 \mathrm{D}$ s-wave and dipolar interaction parameters (see Sec.IIF below). The fourth term on the right-hand-side of (7) introduces the usual mean-field s-wave interaction nonlinearity. The last term introduces the mean-field dipolar nonlinearity derived from the long-range DDI. It is a special integral that is designed to eliminate the singularity in the DDI potential.

Equation (7) is solved numerically using the famous split-step Crank-Nicolson (CN) method [52, 80] in real time. It is first solved in imaginary time to initialize the BEC in the trapping geometry (11) superimposed on which is the NGP (2) without the modulated part, that is

$$
V_{D T}^{(0)}(x)=A \exp \left(-\beta x^{2}\right)
$$

In the second step, the DBEC is driven by the NGP (2) in real time to examine its ensuing dynamics. For further technical details, the reader is referred to Appendix A. The codes used for solving the TDGPE have been written by the group of Antun Balaz in Belgrade and are fully explained in Ref. [52] for recent versions treating DBECs and also Ref. 80] for earlier versions on ordinary BECs. Numerous other TDGPE codes are available by this group [81 87] and have been used extensively.

\section{E. Gross-Pitaevskii Energies}

The Gross-Pitaevskii (GP) energies are as usual evaluated via

$$
\begin{aligned}
& E_{G P}(t)=\int_{-\infty}^{+\infty} d x\left[\left|\frac{\partial \psi(x, t)}{\partial x}\right|^{2}+\right. \\
& {\left[V_{t r}(x)+V_{D T}(x, t)\right]|\psi(x, t)|^{2}+\frac{1}{2} \mathcal{G}_{1 D}|\psi(x, t)|^{4}+} \\
& \left.\frac{2 \pi}{3} \mathcal{G}_{1 d d} \int_{-\infty}^{+\infty} \frac{d k_{x}}{2 \pi} e^{-i k_{x} x}|\widetilde{\psi}(x, t)|^{2} j_{1 D}\left(\tau_{x}\right)|\psi(x, t)|^{2}\right]
\end{aligned}
$$

The time average is then computed using

$$
\left\langle E_{G P}\right\rangle_{t}=\frac{1}{T} \int_{0}^{T} E_{G P}(t) d t .
$$

\section{F. Parameters}

The 3D $s$-wave and dipolar interaction parameters are defined as $\mathcal{G}=4 \pi N a$ and $\mathcal{G}_{d d}=3 N a_{d d}$, where $a$ and $a_{d d}$ are the s-wave and dipolar scattering lengths, and $N$ the number of particles. The interaction parameters acting in $1 \mathrm{D}$ here, $\mathcal{G}_{1 D}$ and $\mathcal{G}_{1 d d}$, are obtained after the reduction of the 3D TDGPE to the 1D form (7). As a result of the latter, the $\mathcal{G}$ and $\mathcal{G}_{d d}$ are divided by a factor $2 \pi d_{\rho}^{2}$ so that

$$
\mathcal{G}_{1 D}=\frac{\mathcal{G}}{2 \pi d_{\rho}^{2}} \quad \text { and } \quad \mathcal{G}_{1 d d}=\frac{\mathcal{G}_{d d}}{2 \pi d_{\rho}^{2}}
$$

where $d_{\rho}$ is the width of the integrated-out wave function in the transverse direction. The $\mathcal{G}_{1 D}$ and $\mathcal{G}_{1 d d}$ are input directly into the code without explicit evaluation via $N$, $a, a_{d d}$, and $d_{\rho}$. These parameters define the strength of the s-wave and dipolar-interaction nonlinearities of the TDGPE. The systems are simulated from $x=-L_{0}$ to $x=+L_{0}$ with $L_{0}=30$ for a harmonic oscillator (HO) trap and quartic trap (QT), and $L_{0}=51.2$ for a box potential. The parameters of the oscillating NGP are $A=-30, \beta=4$, and $\Omega=2 \pi f$ with $f=10$. The values of $\delta A$ applied are 5,10 , and 20. The DBECs are scanned along a range of $\mathcal{G}_{1 d d}$ ranging from 0 to 400 in steps of 2 and for each $\mathcal{G}_{1 d d}$ a run was performed. Values of $\mathcal{G}_{1 D}=50,100,150$ were used for each set of the runs. $\mathcal{G}_{1 D}, \mathcal{G}_{1 d d} L_{0}, a, a_{d d}$, and $d_{\rho}$ are all in units of $a_{h o}$.

\section{G. Signal Energy}

For a time-dependent physical observable $f(t)$, the signal energy $\mathcal{D}$ is formally defined by the integral

$$
\mathcal{D}=\int_{-\infty}^{+\infty}|f(t)|^{2} d t
$$

where $f(t)$ is taken to be the mean-field kinetic energy

$$
E_{k i n}(t)=\frac{1}{2} \int_{-\infty}^{+\infty}\left|\frac{\partial \psi(x, t)}{\partial x}\right|^{2} d x,
$$

$\psi(x, t)$ being the time-dependent wavefunction describing the DBEC. We refer to the signal energy by $\mathcal{D}_{k i n}$ because it is derived from $E_{k i n} . E_{k i n}$ is in units of $\hbar \bar{\omega}$ and $\mathcal{D}_{\text {kin }}$ in $(\hbar \bar{\omega})^{2} \bar{\omega}$. However, since we cannot numerically integrate to infinity, neither timely nor spatially, we limit the integral in Eq.(14) from $t=0$ to some time $T$ 
that is sufficiently long to reveal enough of the dynamical properties, and in Eq. (15) to the length of the simulation grid from $x=-L_{0}$ to $L_{0}$. The usefulness of the signal energy in displaying important properties about excitations in a driven BEC has already been demonstrated in our recent work [2]. There, it has been argued that the signal energy is tantamount to the time-average of the squared amplitude of an oscillating signal describing a dynamic variable. It can be likened to the root-meansquared value of an alternating voltage or current. Since $E_{k i n}(t)$ is found here again to be oscillating with time, it is quite convenient to apply the latter concept to its measurement. The reason for using the kinetic energy stems additonally from the fact that it is an important property of the condensate 88]. Other quantities, such as the potential energy, zero-point energy, and the radial size could also have been used here because they reveal the same PRs with the same properties as obtained for $E_{k i n}$. Further, $E_{k i n}$ has been used in a number of previous examinations [7, 46, 89 92] that further demonstrate its importance.

As far as the experimental measurement of $\mathcal{D}_{\text {kin }}$ is concerned, this can be performed as follows. While the BEC is excited by the focusing red-laser beam of an oscillating intensity, an in-situ recording of density profiles $n(x, t)$ is performed as a function of time by a CCD camera using a nondestructive method as that demonstrated in the experiment of Onofrio et al. 93]. The latter performed a repeated in-situ non-destructive phase-contrast imaging that has been used by Andrews et al. 94] and Ketterle et al. 95]. After the $n(x, t)$ are recorded, they are Fourier-transformed to obtain the corresponding momentum-density distributions $n(\mathbf{p}, t)$ that can be used to compute $E_{k i n}(t)$ via

$$
E_{k i n}(t)=\frac{1}{2 m} \int d p p^{2} n(\mathbf{p}, t) .
$$

Having obtained $E_{k i n}, \mathcal{D}_{k i n}$ is then evaluated easily.

\section{RESULTS}

\section{A. Resonances in a Box Potential}

Figure 1displays $\mathcal{D}_{k i n} / \mathcal{D}_{k i n}^{(0)}$ vs $\mathcal{G}_{1 d d}$ for a few different values of $\mathcal{G}_{1 D}$ and $\delta A$ in a BOX+NGP trap. $\mathcal{D}_{k i n}^{(0)}$ is the maximum signal energy for $\mathcal{G}_{1 D}=50$ at $\delta A=20$ in the range of $\mathcal{G}_{1 d d}$ considered and is used for normalization of the signals in all frames. Several PRs are discovered whose amplitudes decline with increasing values of $\mathcal{G}_{1 d d}$. This is in line with Refs. [75, 96], where it has been found that the DDI reduce the amplitude of DBEC dynamics. An increase in the DDI causes therefore a weaker response to the oscillating NGP as it turns out that the repulsive DDI reduce the depth of the effective mean-field potential (see Sec. IIID below) and with it the occupancy of the NGP so that lesser particles are excited.

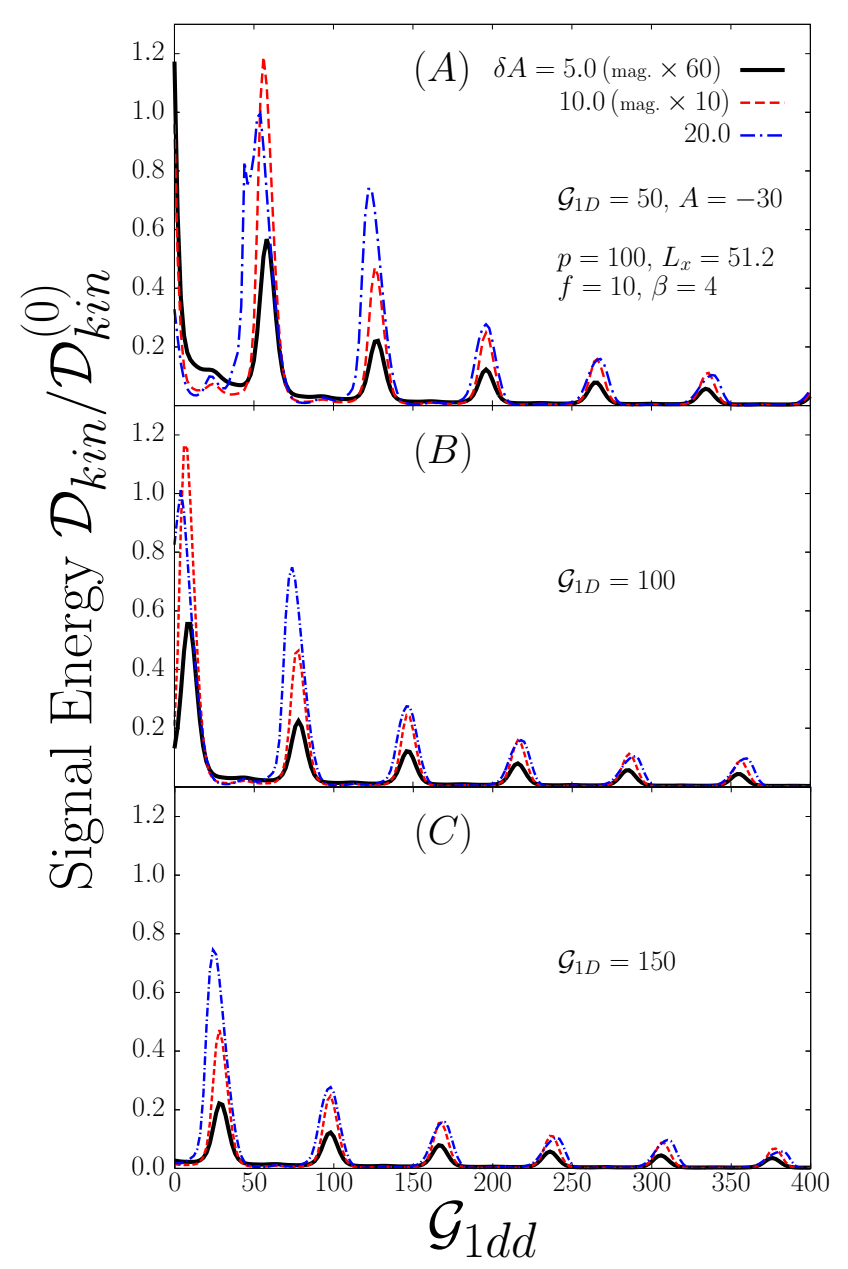

FIG. 1: Signal energy $\mathcal{D}_{k i n}$ of a driven one-dimensional DBEC in a box as a function of the dipolar interaction parameter $\mathcal{G}_{1 d d}$ [Eq. 13)]. $\mathcal{D}_{\text {kin }}$ is normalized by $\mathcal{D}_{\text {kin }}^{(0)}$, the maximum of $\mathcal{D}_{k i n}$ at $\delta A=20$ in the $\mathcal{G}_{1 d d}$-range considered. $\mathcal{G}_{1 D}$ is the $s$-wave interaction parameter [Eq.(13)]. The box is generated from Eq.(11) using $L_{x}=51.2$ and $p=100$. The DBEC is excited by an NGP whose principal depth $A$ is periodically modulated by an amplitude $\delta A$ via Eq.(2) with $A=-30$, $f=10$, and $\beta=4$. Various values are used for $\delta A$ and $\mathcal{G}_{1 D}$. Frame $(A)$ and thick-solid line: $\mathcal{D}_{k i n}$ at $\mathcal{G}_{1 D}=50$ with $\delta A=$ 5. The signal is magnified (mag.) by 60 times $(\times)$ to enable comparison with other cases; thin-dashed line: $\delta A=10.0$ (mag. $\times 10)$; and dashed-dotted line: 20.0 (no mag.). Frames $(B, C)$ : as in $(A)$; but for $\mathcal{G}_{1 D}=100$ and 150 , respectively. $A$ and $\delta A$ are in units of $\hbar \bar{\omega} ; L_{x}, \mathcal{G}_{1 D}, \mathcal{G}_{1 d d}$ in $a_{h o}$, whereas $\beta$ in $a_{h o}^{-2} . \mathcal{D}_{k i n}$ and $\mathcal{D}_{k i n}^{(0)}$ are in $(\hbar \bar{\omega})^{2} \bar{\omega}$.

Qualitatively, the same features are observed for all values of $\mathcal{G}_{1 D}$ in Fig. 1. Notably, an increase in $\mathcal{G}_{1 D}$ shifts the whole spectrum backwards (to the left) keeping the same distance $\Delta \mathcal{G}_{1 d d}$ between each pair of peaks, demonstrating that $\Delta \mathcal{G}_{1 d d}$ is not influenced by $\mathcal{G}_{1 D}$. For example, the third peak from the left in frame $(A)$ at $\mathcal{G}_{1 d d}$ $\sim 200$ is shifted backwards by an amount of 50 in frame $(B)$ from its position in $(A)$, and by an amount 100 in 


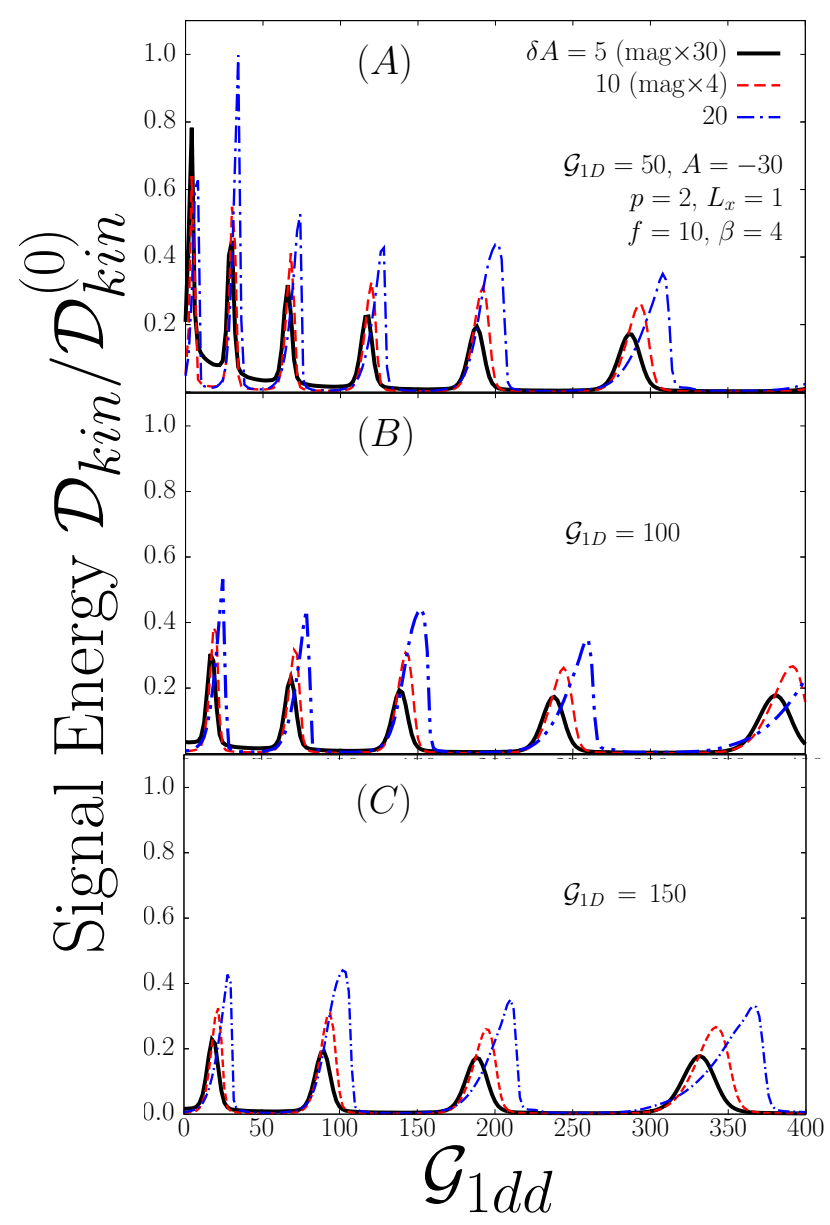

FIG. 2: As in Fig. 1 but for an HO trap with $p=2, L_{x}=1$, magnifications of $\times 30$ for $\delta A=5$ and $\times 4$ for $\delta A=10$, and $\mathcal{D}_{\text {kin }}^{(0)}$ is the maximum of $\mathcal{D}_{\text {kin }}$ at $\delta A=20$ in frame $(A) . A$ and $\delta A$ are in units of $\hbar \bar{\omega} ; L_{x}, \mathcal{G}_{1 D}, \mathcal{G}_{1 d d}$ in $a_{h o}$, whereas $\beta$ in $a_{h o}^{-2}$. $\mathcal{D}_{k i n}$ and $\mathcal{D}_{k i n}^{(0)}$ are in $(\hbar \bar{\omega})^{2} \bar{\omega}$.

frame $(C)$. That is, an increase of $\mathcal{G}_{1 D}$ by 50 causes all the resonance positions to shift backwards by the same value of 50, (similarly for an increase by 100) being a rather unprecedented and remarkable feature. Thus there seems to be no effect for the interplay between $s$-wave interactions and DDI on the principle features of the spectrum of $\mathcal{D}_{\text {kin }}$ in a box potential. This demonstrates that $s$-wave interactions and DDI work similarly in determining the positions of PRs. The above results reveal information about the energy-level structure of the DBEC and shape of the trapping potential. The equidistance of peaks mirrors the confinement homogeneity, i.e. the flatness of the box. This can be particularly concluded from a comparison with the $\mathrm{HO}$ potential in the next section.

The reason for the PRs and the shift of their positions with $\mathcal{G}_{1 D}$ are discussed in Sec.IV basing on well-known theoretical methods: the Wenzel-KramerBrillouin (WKB) approximation, time-independent and time-dependent perturbation theory, and LVM. Most im- portantly, it is shown that the PRs arise whenever the time-averaged energy of the DBEC closely matches one of the energy levels of the trap+NGP.

\section{B. Resonances in a Harmonic Trap}

In Fig. 2. PRs are also encountered in an $\mathrm{HO}+\mathrm{NGP}$ trap, except that the separation $\Delta \mathcal{G}_{1 d d}$ is not uniform as in the box but rather increases with $\mathcal{G}_{1 d d}$. Henceforth, this mirrors confinement inhomogeneity. Further, this reveals the role of the interplay between trapping geometry and DDI in determining the PR energies. A uniform trap leads to equidistant whereas a nonuniform one to non-equidistant PRs along the $\mathcal{G}_{1 d d}$ axis. What is peculiar though, is that the shift of the whole spectrum as a result of changing $\mathcal{G}_{1 D}$ is also observed here in the same uniform manner as reported in Fig. 1 Increasing $\mathcal{G}_{1 D}$ by 50 causes the PR peaks to shift backwards by 50 along the $\mathcal{G}_{1 d d}$ axis.

\section{Resonances in a Quartic Trap}

In Fig. 3 for a QT+NGP, there is only one welldefined PR at $\delta A=5$ in the same range of $\mathcal{G}_{1 d d}$ considered. At the larger $\delta A$, a disordered excitation pattern arises. Compared to Figs. 1 and 2, this is rather surprising since a pattern similar to that for the $\mathrm{HO}+\mathrm{NGP}$ trap had been anticipated. Thus for a QT at stronger driving quite a larger number of modes is excited than in the $\mathrm{HO}$ trap and box. As such, there exist trapping geometries that under certain conditions do not support ordered PR patterns. Once again it can be seen that the geometry of the trap strongly influences the PR phenomenon and its pattern. Therefore, it can be used to control the PRs. As in the previous figures, an increase of $\mathcal{G}_{1 D}$ by 50 causes the whole PR spectrum to shift by 50 along the $\mathcal{G}_{1 d d}$ axis.

\section{Effective Potential}

To this end, it is useful to state the reason for the decline of the amplitude of a PR with $\mathcal{G}_{1 d d}$ as observed in Figs. 1 1and 2. This decline is attributed to a change in the depth of the time-averaged effective potential given by

$$
\begin{aligned}
& \left\langle V_{\text {eff }}(x)\right\rangle_{t}=\left\langle V_{D T}(x, t)+\mathcal{G}_{1 D}|\psi(x, t)|^{2}+\right. \\
& \left.\frac{4 \pi}{3} \mathcal{G}_{1 d d} \int_{-\infty}^{+\infty} \frac{d k_{x}}{2 \pi} e^{-i k_{x} x} j_{1 D}\left(\tau_{x}\right)\left|\widetilde{\psi}\left(k_{x}, t\right)\right|^{2}\right\rangle+V_{t r}(x),
\end{aligned}
$$

with 


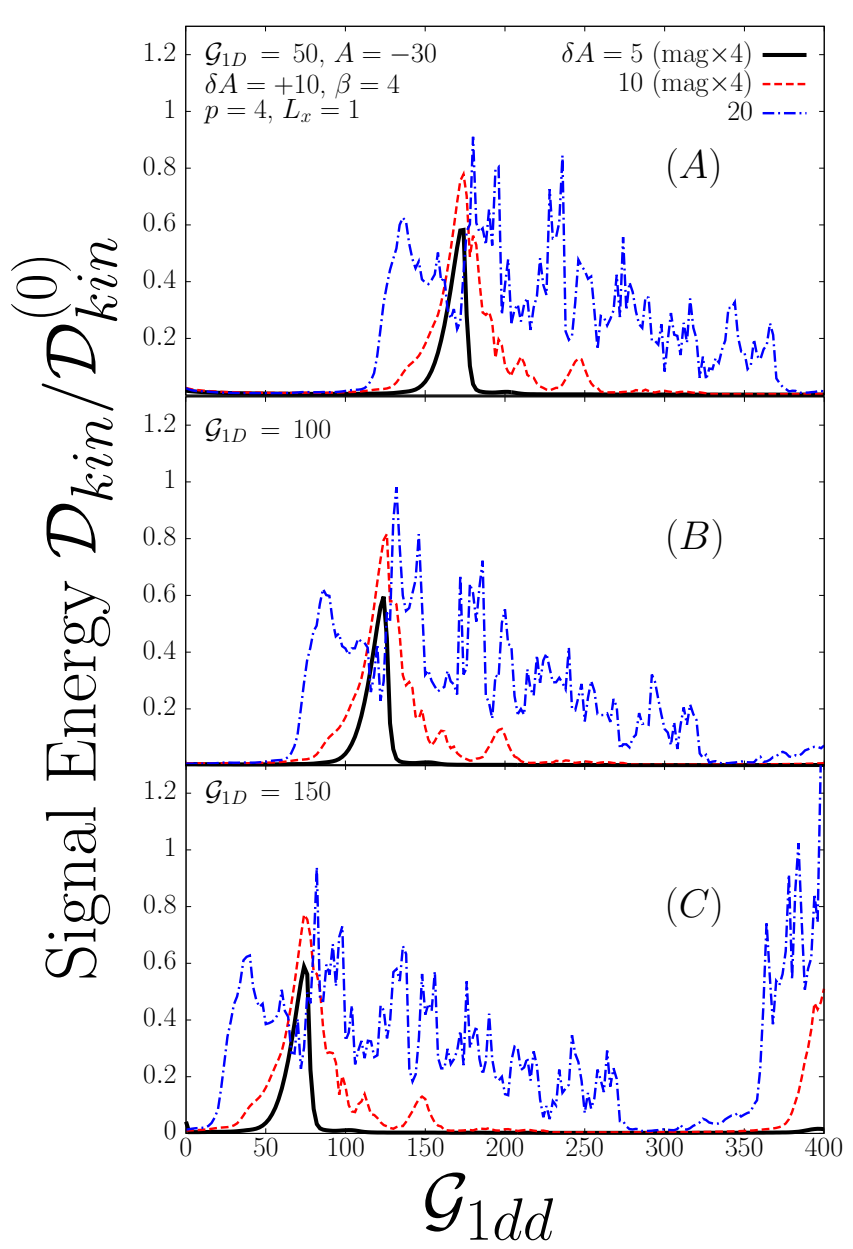

FIG. 3: As in Fig. 11 but for a QT with $p=4, L_{x}=1$, magnification of $\times 4$ for $\delta A=5$ and 10 , and $\mathcal{D}_{k i n}^{(0)}$ is a normalization factor. $A$ and $\delta A$ are in units of $\hbar \bar{\omega} ; L_{x}, \mathcal{G}_{1 D}, \mathcal{G}_{1 d d}$ in $a_{h o}$, whereas $\beta$ in $a_{h o}^{-2}$. $\mathcal{D}_{\text {kin }}$ and $\mathcal{D}_{k i n}^{(0)}$ are in $(\hbar \bar{\omega})^{2} \bar{\omega}$

$$
\langle\cdots\rangle=\frac{1}{T} \int_{0}^{T}(\cdots) d t
$$

$T$ being the total simulation time. Fig. 4 $(A)$ shows as an example $\left\langle V_{\text {eff }}(x)\right\rangle_{t}$ for the HO+NGP of Fig. $2(A)$. Fig. 4( $B)$ displays the minimum of $\left\langle V_{\text {eff }}(x)\right\rangle_{t}$ at $x=0$ for previous systems in Figs. 1 and 2 as a function of the PR values of $\mathcal{G}_{1 d d} .\left\langle V_{\text {eff }}(x)\right\rangle_{t}$ becomes shallower as $\mathcal{G}_{1 d d}$ rises causing lesser particles to occupy the NGP. Hence, lesser particles contribute to the strength of the excitations as they are thrown out of the NGP by its oscillating depth thereby causing the drop in the PR amplitudes $\mathcal{D}_{\text {kin }}$. Notably, $\left\langle V_{\text {eff }}(x)\right\rangle_{t}$ closely follows the spatial shape of the NGP and it can be therefore argued that the influence of the NGP on the DBEC is reduced as $\left\langle V_{e f f}(x)\right\rangle_{t}$ becomes shallower. Further the depth $\left\langle V_{\text {eff }}(x)\right\rangle_{t}$ being controlled by $\mathcal{G}_{1 d d}$ plays also a decisive role in determining the PR energies.
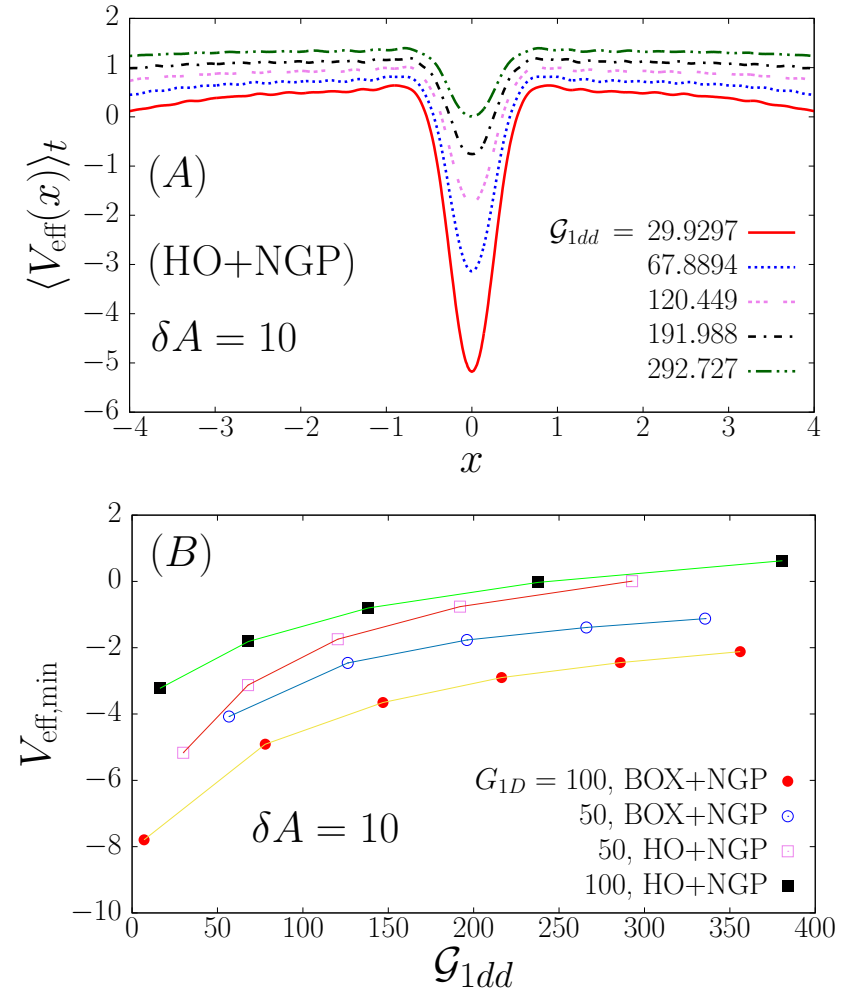

FIG. 4: Frame $(A)$ : Time-averaged effective potential $\left\langle V_{\text {eff }}(x)\right\rangle_{t}[$ Eq.(17)] for the HO+NGP in Fig. 2( $A)$ at $\delta A=10$ and the indicated resonant values of $\mathcal{G}_{1 d d}$. Solid line: 29.9297; dotted line: 67.8894; triple-dotted line: 120.449; dasheddotted line: 191.988; dashed-double-dotted line: 292.727. Frame $(B)$ : Minima $V_{\text {eff,min }}=\left\langle V_{e f f}(0)\right\rangle_{t}$ at the PR values of $\mathcal{G}_{1 d d}$ for some of the systems presented in Figs. 1 and 2 at $\delta A=10$ : Solid circles: $\mathrm{BOX}+\mathrm{NGP}$ at $\mathcal{G}_{1 D}=100$; open circles: same at 50; open squares: $\mathrm{HO}+\mathrm{NGP}$ at $\mathcal{G}_{1 D}=50$; solid squares: same at 100 . $\left\langle V_{\text {eff }}(x)\right\rangle_{t}, V_{\text {eff,min }}, A$, and $\delta A$ are in units of $\hbar \bar{\omega}$, whereas $L_{x}, x, \mathcal{G}_{1 D}$, and $\mathcal{G}_{1 d d}$ in units of $a_{h o}$, and $\beta$ in $a_{h o}^{-2}$.

\section{ORIGINS OF THE PARAMETRIC RESONANCES}

The reasons for the appearance of the PRs at certain values of $\mathcal{G}_{1 d d}$ are deeply encrypted in the numerical solutions of the TDGPE and hard to decipher. Therefore, there is no other way to circumvent this problem but to seek qualitative explanations basing on other models and methods, such as LVM [7, 45 51, 97], the WKB approximation, as well as time-dependent and time-independent perturbation theory [98, 99]. It should be noted that LVM has been applied earlier to treat the same HO+NGP system as the present one, but without DDI [7]. The latter investigation also demonstrated the presence of PRs although in a different framework. Astonishingly, by using LVM in the present work it has been possible to generate a few resonances in a manner similar to those obtained in Figs. 1- 3, The LVM analysis 
of these PRs can be used in a qualitative manner to cast more light on them and to reach a better understanding of their origin. The time-averaged GP energies of some of our systems at PR were matched to energy levels calculated by using the perturbation theory corresponding to either the $\mathrm{HO}+\mathrm{NGP}, \mathrm{BOX}+\mathrm{NGP}$, or $\mathrm{QT}+\mathrm{NGP}$ traps. This enables us to characterize their level structure. By applying time-dependent perturbation theory, it has been demonstrated that each PR corresponds to a certain transition between an energy level $m$ of the trap and the energy level $n$ to which the PR has been matched, identified by the maximum transitional probability between a set of other probabilities to this state $n$.

\section{A. LVM analysis}

\section{Euler-Lagrange Differential Equation}

The Euler-Lagrange equation for the dynamics of the width $w(t)$ of the DBEC in a 1D harmonic trap can be found in Ref. [45]. We thus add to this LVM equation the contribution coming from the NGP with oscillating depth [Eq.(2)]. Further, using our definitions for $\mathcal{G}_{1 d d}$ and $\mathcal{G}_{1 D}$ [Eq.(13)], this LVM equation becomes

$$
\begin{aligned}
& \ddot{w}+w=\frac{1}{w^{3}}+\frac{\mathcal{G}_{1 D}}{\sqrt{2 \pi} w^{2}}- \\
& \frac{\beta[A+\delta A \cos (\Omega t)] w}{\left(1+\beta w^{2}\right)^{3 / 2}}-2 \sqrt{2 \pi} \frac{\mathcal{G}_{1 d d}}{3 w^{2}} c(\kappa)
\end{aligned}
$$

with $\kappa=d_{\rho} / w$,

$$
c(\kappa)=\frac{1+10 \kappa^{2}-2 \kappa^{4}-9 \kappa^{2} d(\kappa)}{\left(1-\kappa^{2}\right)^{2}}
$$

and

$$
d(\kappa)=\frac{\operatorname{arctanh} \sqrt{1-\kappa^{2}}}{\sqrt{1-\kappa^{2}}}
$$

The width at time $t=0$ is used as an initial condition for solving the LVM equation (19) and is taken to be the value $w_{0}=w(t=0)$ at which the DBEC is in equilibrium. As in Refs. 7, 46], $w_{0}$ is obtained by solving the equation

$w_{0}-\frac{1}{w_{0}^{3}}-\frac{\mathcal{G}_{1 D}}{\sqrt{2 \pi} w_{0}^{2}}+\frac{A \beta w_{0}}{\left(1+\beta w_{0}^{2}\right)^{3 / 2}}+2 \sqrt{2 \pi} \frac{\mathcal{G}_{1 d d}}{3 w_{0}^{2}} c\left(\kappa_{0}\right)=0$,

at $\kappa_{0}=d_{\rho} / w_{0}$ that is gotten from Eq. 19 by setting $\delta A=0$ and $\ddot{w}=0$.

\section{Frequency of Breathing Mode}

In this section, we revisit the frequency of the breathing mode explored earlier in some of our publications 7, 46], except that this time the effects of the DDI are added. As before, the breathing-mode frequency can be obtained by a linearization of Eq.(19) via $w(t)=$ $w_{0}+\delta w(t)$. However, to avoid mathematical complexities, it is easier to equivalently take the differential of both sides of (19) with respect to $w$ at $w_{0}$. Consequently, one gets

$$
\delta \ddot{w}+\Omega_{B}^{2} \delta w=0
$$

with $\Omega_{B}^{2}$ given by

$$
\begin{aligned}
\Omega_{B}^{2}(t)= & 1+\frac{3}{w_{0}^{4}}+\sqrt{\frac{2}{\pi}} \frac{\mathcal{G}_{1 D}}{w_{0}^{3}}+ \\
& \frac{[A+\delta A \cos (\Omega t)] \beta\left(1-2 \beta w_{0}^{2}\right)}{\left(1+\beta w_{0}^{2}\right)^{5 / 2}}+ \\
& 2 \sqrt{2 \pi} \frac{\mathcal{G}_{1 d d}}{3 w^{2}}\left[\left.\frac{\partial c(\kappa)}{\partial w}\right|_{w_{0}}-\frac{2}{w} c\left(\kappa_{0}\right)\right] .
\end{aligned}
$$

The term between brackets yields

$$
\begin{aligned}
& \left.\frac{\partial c(\kappa)}{\partial w}\right|_{\kappa_{0}}-\frac{2}{w_{0}} c\left(\kappa_{0}\right)= \\
& \frac{\kappa_{0}}{d_{\rho}\left(1-\kappa_{0}^{2}\right)^{3}}\left(-2-51 \kappa_{0}^{2}+12 \kappa_{0}^{4}-4 \kappa_{0}^{6}+\right. \\
& \left.\frac{9 \kappa_{0}^{2}\left(\kappa_{0}^{2}+4\right)}{\sqrt{1-\kappa_{0}^{2}}} \operatorname{arctanh} \sqrt{1-\kappa_{0}^{2}}\right) .
\end{aligned}
$$

Again, it can be concluded that an imaginary value of $\Omega_{B}$ is obtained if

$$
\begin{aligned}
& 1+\frac{3}{w_{0}^{4}}+\sqrt{\frac{2}{\pi}} \frac{\mathcal{G}_{1 D}}{w_{0}^{3}}< \\
& -\frac{[A+\delta A \cos (\Omega t)] \beta\left(1-2 \beta w_{0}^{2}\right)}{\left(1+\beta w_{0}^{2}\right)^{5 / 2}} \\
& -2 \sqrt{2 \pi} \frac{\mathcal{G}_{1 d d}}{3 w_{0}^{2}}\left[\left.\frac{\partial c(\kappa)}{\partial w}\right|_{w_{0}}-\frac{2}{w_{0}} c\left(\kappa_{0}\right)\right],
\end{aligned}
$$

that leads to a damping of the DBEC oscillations.

\section{LVM kinetic energy}

The LVM kinetic energy is given by [7, 45, 46]

$$
e_{k i n}(t)=\frac{1}{2}[\dot{w}(t)]^{2}+\frac{1}{2[w(t)]^{2}}
$$


and is used now for $f(t)$ in Eq.(14) for evaluating $\mathcal{D}_{\text {kin }}$ and checking the possibility of generating PRs by the LVM equation (19).

\section{LVM Results}

The LVM equation (19) is solved numerically with Mathematica over the same range of $\mathcal{G}_{1 d d}$ as in Sec. III using similar parameters for $\mathcal{G}_{1 D}, A, \delta A, L_{x}$, and $\beta$, except for $\Omega$ which is set to other values for the best response of the system to changes in $\mathcal{G}_{1 d d}$. For example, an integer value for $\Omega$ yields resonant behavior in $w(t)$ and $\mathcal{D}_{\text {kin }}$ contrary to a real value [7]. In this regard, it should be emphasized that we principally aim at a qualitative comparison with TDGPE that would help us in explaining the PRs of Sec. III.

In Fig. 5 the signal energy $\mathcal{D}_{k i n}$ obtained from $e_{k i n}(t)$, where the latter is given by (27), is graphed as a function of $\mathcal{G}_{1 d d}$ and demonstrates that LVM surprisingly generates PRs along the same lines as the ones displayed in Sec. III. Another astonishing result is that these resonances are shifted in their positions along the $\mathcal{G}_{1 d d}$ axis when $\mathcal{G}_{1 D}$ is changed. This is similar to what has been reported earlier in Figs. 1- 3 of the TDGPE results, except that in LVM they shift in the opposite direction and their amplitudes and shapes in Fig. 5 change somewhat because of this shift. This opposite behavior is an artefact of the model arising from the fact that LVM relies on the variational Gaussian Ansatz [45 47]

$$
\psi(x)=\frac{1}{\pi^{1 / 4} w} e^{-\frac{x^{2}}{2 w^{2}}+i \beta x^{2}}
$$

to evaluate the mean-field Lagrangian [7, 45 51]. This Ansatz is much less flexible than the numerical solution to the TDGPE resulting in this different behavior. It should further be iterated that it is mostly suitable for BECs in an HO trap and thus needs to be developed for other types of traps. Nevertheless the discovery of the LVM PRs as presented in Fig. 5 strongly substantiates our results on the TDGPE PRs and supports our claims that they are an inherent feature in the DBECs, and not just a result of some other influence such as noise or numerical chaos.

Figure 6 displays the time-average of $\Omega_{B}(t)$ [Eq.(24)], that is $\left\langle\Omega_{B}\right\rangle_{t}$ graphed against $\mathcal{G}_{1 d d}$ for various $\mathcal{G}_{1 D}$. For a larger $\mathcal{G}_{1 D}$, the height of the curve drops. This can be connected to the PR shifting to larger resonant values of $\mathcal{G}_{1 d d}$ in Fig. 囵 Inspecting Eq.(24), one can see that the term proportional to $\mathcal{G}_{1 D}$ is linearly added to that proportional to $\mathcal{G}_{1 d d}$. Thus when $\left\langle\Omega_{B}\right\rangle_{t}$ is changed as a result of varying $\mathcal{G}_{1 D}$, the position of the corresponding $\mathrm{PR}$ is updated.

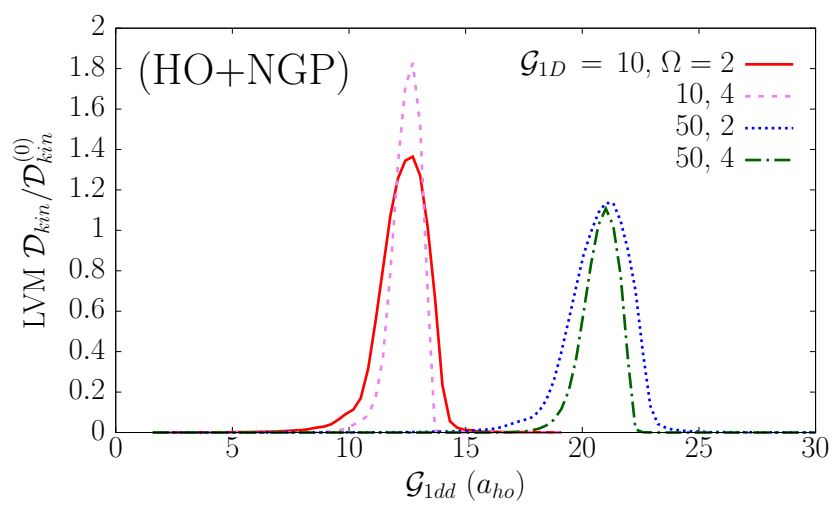

FIG. 5: Parametric resonances generated by the EulerLagrange differential equation (19) for a 1D DBEC in the same HO+NGP trap of Fig. 2 The graph displays the signal energy $\mathcal{D}_{k i n}$ of the LVM kinetic energy $e_{k i n}(t)$ [Eq.(27)] versus the DDI interaction parameter $\mathcal{G}_{1 d d}$. $\mathcal{D}_{\text {kin }}$ is normalized by $\mathcal{D}_{\text {kin }}^{(0)}=1 \times 10^{6}$. Different values for $\mathcal{G}_{1 D}$ and $\Omega$ are considered: 10 and 2 , respectively (solid line); 10, 4 (dashed line); 50, 2 (dotted line); and 50, 4 (dashed-dotted line). The initial conditions used in solving the Euler-Lagrange equation (19) at time $t=0$ are the initial "speed" $\dot{w}=\dot{w}(0)=0$ and the values of the equilibrium $w_{0}=w(0)$ satisfying Eq.(22) at each $\mathcal{G}_{1 d d}$ with the value of $d_{\rho}$ in $\kappa=d_{\rho} / w$ set to 0.6 . $\mathcal{G}_{1 D}$, $\mathcal{G}_{1 d d}$, and $L_{x}$ are in units of $a_{h o}$ whereas $A$ in $\hbar \bar{\omega}$ and $\beta$ in $a_{h o}^{-2}$. $\mathcal{D}_{\text {kin }}$ and $\mathcal{D}_{\text {kin }}^{(0)}$ are in units of $(\hbar \bar{\omega})^{2} \bar{\omega}$.

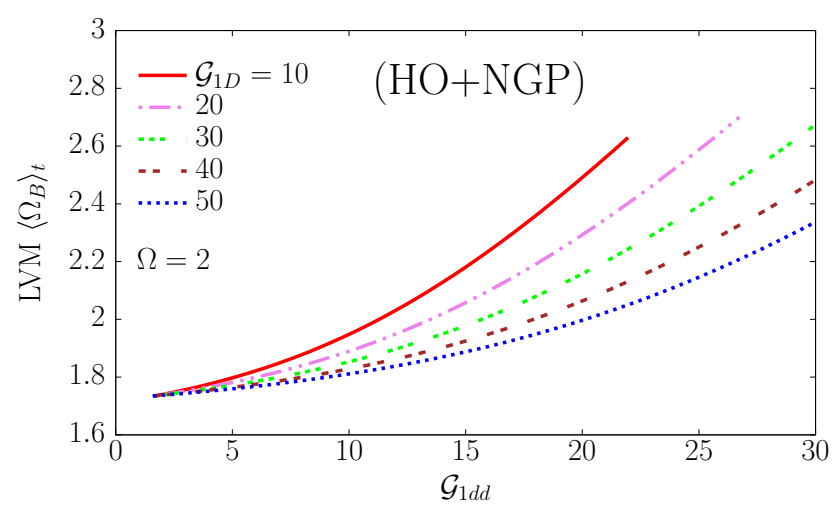

FIG. 6: As in Fig. 5 but for the time average $\left\langle\Omega_{B}\right\rangle_{t}$ of the LVM breathing-mode frequency $\Omega_{B}(t)$ given by [Eq. (24)] at various values of $\mathcal{G}_{1 D}$. Solid line: $\mathcal{G}_{1 D}=10$; dashed-doubledotted line: 20; triple-dotted line: 30; double-dotted line: 40; dotted line: $50 . \Omega_{B}(t)$ is in units of $\bar{\omega}$.

\section{B. Energy Levels of the HO+NGP Trap via Perturbation Theory}

The goal of this section is to demonstrate that the GP time-averaged total energies $\left\langle E_{G P}\right\rangle_{t}[\mathrm{Eq}$. [12)] at which the driven DBEC resonates can be generated from first and second-order perturbation theory thereby revealing a certain ELS via the quantum numbers $n$ corresponding to $\left\langle E_{G P}\right\rangle_{t}$. The HO+NGP is considered here first. Within this context, the NGP is taken to be a small per- 
TABLE I: Match of the time averaged GP energies $\left\langle E_{G P}\right\rangle_{t}$ [Eq. [12)] with the energy levels $E_{n}$ obtained from second-order perturbation theory. The system considered here is the HO+NGP trap in Fig. 2( $A)$ for $\delta A=10$. The $E_{n}$ are obtained from Eqs. 29 33). From left to right the table lists $\mathcal{G}_{1 d d},\left\langle E_{G P}\right\rangle_{t}, E_{n}$ at quantum state $n$, the number of states $M$ found for the second-order correction (31), and the difference between the matched energies. $\left\langle E_{G P}\right\rangle_{t}$ and $E_{n}$ are in units of $\hbar \bar{\omega}$, and $\mathcal{G}_{1 d d}$ in $a_{h o}$.

\begin{tabular}{|c|c|c|c|c|c|}
\hline $\mathcal{G}_{1 d d}$ & $\left\langle E_{G P}\right\rangle_{t}$ & $E_{n}$ & $n$ & $M$ & $\left|\left\langle E_{G P}\right\rangle_{t}-E_{n}\right|$ \\
\hline 29.9297 & 2.67631 & 2.68257 & 4 & 10 & 0.00626 \\
67.8894 & 5.79083 & 5.77971 & 7 & 20 & 0.01112 \\
120.449 & 9.62129 & 9.5773 & 10 & 21 & 0.04399 \\
191.988 & 15.1178 & 15.1395 & 13 & 11 & 0.02170 \\
292.727 & 18.3912 & 18.3361 & 18 & 21 & 0.05510 \\
\hline \hline
\end{tabular}

turbation within a large HO trap. This is in view of the fact that the size of our simulated system is $60\left(a_{h o}\right)$ casting a very a high potential energy at the trap edges of the order of $\sim 450(\hbar \omega)$. Therefore, it is reasonable to apply time-independent perturbation theory to calculate the energy levels of the DBEC. The first order correction to the energy is thus

$$
\begin{aligned}
E_{n}^{(1)} & =\left\langle\phi_{n}(x)\left|V_{D T}(x)\right| \phi_{n}(x)\right\rangle \\
& =\frac{A}{\sqrt{\pi} 2^{n} n !} \int_{-\infty}^{+\infty} d x H_{n}^{2}(x) e^{-(\beta+1) x^{2}}
\end{aligned}
$$

where $\phi_{n}(x)$ are the HO functions being the solutions to the non-interacting Schrödinger equation with an $\mathrm{HO}$ potential and are given by

$$
\phi_{n}(x)=\frac{1}{\sqrt{2^{n} n ! \sqrt{\pi}}} H_{n}(x) e^{-x^{2} / 2} . \quad(n=1,2,3, \cdots)
$$

The second-order correction arising from a number of levels $M$ is thus

$$
\begin{aligned}
E_{n}^{(2)}= & \sum_{\substack{k \\
k \neq n}}^{M} \frac{\left|\left\langle\phi_{n}(x)\left|V_{D T}(x)\right| \phi_{k}(x)\right\rangle\right|^{2}}{E_{k}^{(0)}-E_{n}^{(0)}} \\
= & \frac{A^{2}}{\pi} \sum_{\substack{k \\
k \neq n}}^{M} \frac{1}{2^{n+k} n ! k !(k-n)} \times \\
& \left|\int_{-\infty}^{+\infty} d x H_{n}(x) H_{k}(x) e^{-(\beta+1) x^{2}}\right|^{2} .
\end{aligned}
$$

The above Eqs.(29) and (31) can be evaluated numerically by Mathematica. The energy level $E_{n}$ of the DBEC in the $\mathrm{HO}+\mathrm{NGP}$ is then the sum of the latter corrections plus the unperturbed energy level

$$
E_{n}^{(0)}=\left(n+\frac{1}{2}\right)
$$

such that

$$
E_{n}=E_{n}^{(0)}+E_{n}^{(1)}+E_{n}^{(2)} .
$$

Table I lists for example the $\left\langle E_{G P}\right\rangle_{t}$ of the DBEC at the resonant values of $\mathcal{G}_{1 d d}$ for the system in Fig. 2(A) at $\delta A=10$ with corresponding values $E_{n}$ obtained from Eqs.(29) (33). The values of $\left\langle E_{G P}\right\rangle_{t}$ and $E_{n}$ agree very well after matching and consequently an analytical ELS can be deduced.

It makes sense to state that when $\left\langle E_{G P}\right\rangle_{t}$ matches with an $E_{n}$ the PR occurs. Therefore, a Green's function of the form

$$
G(E) \sim \frac{1}{E-E_{n}+i \Gamma}
$$

could be proposed that accounts for these PRs, where $\Gamma$ is a width and $E=\left\langle E_{G P}\right\rangle_{t}$. The latter $\left\langle E_{G P}\right\rangle_{t}$ increase with $\mathcal{G}_{1 d d}$ while $\left|\left\langle V_{\text {eff }}(0)\right\rangle\right|$ [see Sec. IIID and Eq. [17]] drops with it. The quantum number $n$ rises as well indicating that the PRs shift to higher frequencies. In fact, this can be related to the results of the experiment of Balik et al. [1] who excited PRs via a $\mathrm{CO}_{2}$ laser with an intensity modulated via a depth $h$. They showed that a reduction in $h$ shifts the PRs to higher frequencies. Our findings above are analogous to theirs for this case.

\section{Energy Levels in a BOX+NGP trap via Perturbation Theory}

The goal of this section is the same as the prior one, but for the box potential. The wave function $\phi_{n}(x)$ for a $1 \mathrm{D}$ box of length $2 L_{x}$ is appropriately

$$
\phi_{n}(x)=\frac{1}{\sqrt{L_{x}}} \cos \left(\frac{n \pi x}{2 L_{x}}\right), \quad(n=1,3,5, \cdots)
$$

again being the solution to the box-potential Schrödinger equation in the noninteracting case. Similar to Eqs.(29) and (31) this yields 
TABLE II: As in Table 1 but for the BOX+NGP in Fig. [ $(A)$ with the corrections to the energies obtained via $\phi_{n}(x)$ given by Eq.(34). $\left\langle E_{G P}\right\rangle_{t}$ and $E_{n}$ are in units of $\hbar \bar{\omega}$, and $\mathcal{G}_{1 d d}$ in $a_{h o}$.

\begin{tabular}{|c|c|c|c|c|c|}
\hline $\mathcal{G}_{1 d d}$ & $\left\langle E_{G P}\right\rangle_{t}$ & $E_{n}$ & $n$ & $M$ & $\left|\left\langle E_{G P}\right\rangle_{t}-E_{n}\right|$ \\
\hline 7.0 & 8.00596 & 7.96441 & 131 & 129 & 0.04155 \\
78.0 & 5.44211 & 5.43156 & 109 & 101 & 0.01055 \\
146.882 & 3.55976 & 3.56896 & 89 & 81 & 0.00920 \\
216.331 & 3.27785 & 3.27527 & 87 & 21 & 0.00258 \\
285.841 & 3.30829 & 3.31255 & 85 & 81 & 0.00426 \\
356.060 & 2.23922 & 2.25459 & 73 & 103 & 0.01537 \\
\hline
\end{tabular}

$$
E_{n}^{(1)}=\frac{A}{L_{x}} \int_{-L_{x}}^{+L_{x}} d x\left[\cos \left(\frac{n \pi x}{2 L_{x}}\right)\right]^{2} e^{-\beta x^{2}}
$$

and

$$
\begin{aligned}
& E_{n}^{(2)}=\frac{8 A^{2}}{\pi^{2}} \sum_{\substack{k \\
k \neq n}}^{M} \frac{1}{\left(k^{2}-n^{2}\right)} \times \\
& \left|\int_{-L_{x}}^{+L_{x}} d x \cos \left(\frac{n \pi x}{2 L_{x}}\right) \cos \left(\frac{k \pi x}{2 L_{x}}\right) e^{-\beta x^{2}}\right|^{2},
\end{aligned}
$$

where the unperturbed energies are given by

$$
E_{n}^{(0)}=\frac{n^{2} \pi^{2}}{8 L_{x}^{2}}
$$

(which are in trap units). Accordingly, Table II lists the same quantities as in Table I but for the BOX+NGP. It is found again that perturbation theory is able to reproduce the GP energies at which the PRs occur. It makes sense also here to state that when $\left\langle E_{G P}\right\rangle_{t}$ matches $E_{n}$ a PR arises. Notably, the PRs correspond to very high energy levels $n$ because the size of the system $2 L_{x}$ is quite large $\left(L_{x}=51.2\right)$ yielding small differences in the $E_{n}$ and thereby a large density of states. However, the behavior of $\left\langle E_{G P}\right\rangle_{t}$ with $\mathcal{G}_{1 d d}$ is opposite to that of the $\mathrm{HO}+\mathrm{NGP}$. The reduction in the depth of the effective potential with $\mathcal{G}_{1 d d}$ causes the PR energies to drop shifting them to lower frequencies instead. Thus the DDI in the case of a BOX +NGP cannot prevent the bosons from falling deeper into the NGP. But why does this happen for the box and not the HO? Two reasons come to mind: (1) the density in the BOX+NGP is lower than in the $\mathrm{HO}+\mathrm{NGP}$ as a result of which the dipolar nonlinearity is weaker in the box; (2) the quantum pressure in the $\mathrm{HO}$ trap is larger than in the box. This finding, in conjunction with the one in the previous section, demonstrates the role of an interplay between the trapping geometry and interactions in defining the positions of the PRs in the spectrum of $\mathcal{D}_{k i n}$.

\section{Energy Levels in a QT+NGP via Perturbation Theory}

The energy levels of a general power-law trap

$$
V_{t r}(x)=\alpha|x|^{\nu}
$$

can be obtained from WKB theory as described in advanced quantum mechanics textbooks [99] and yields (by setting $\hbar=m=1$ )

$$
E_{n}^{(0)}=\alpha\left[\left(n+\frac{1}{2}\right) \sqrt{\frac{\pi}{2 \alpha}} \frac{\Gamma\left(\frac{1}{\nu}+\frac{3}{2}\right)}{\Gamma\left(\frac{1}{\nu}+1\right)}\right]^{\frac{2 \nu}{\nu+2}}
$$

The left and right turning points $a$ and $b$, respectively, are obtained from

$$
a=-b=-\left(\frac{E_{n}^{(0)}}{\alpha}\right)^{1 / \nu}
$$

where in the present case $\alpha=1 / 2$ and $\nu=4$. The WKB wave functions are determined by the usual matching of the solutions at the turning points and read

$$
\phi_{n}(x)=\left\{\begin{array}{cc}
\frac{R}{\sqrt{\kappa_{n}(x)}} e^{-\int_{x}^{a} \kappa_{n}(y) d y} ; & x \ll a \\
\frac{2 R}{\sqrt{k_{n}(x)}} \sin \left[\int_{x}^{b} k_{n}(y) d y+\frac{\pi}{4}\right] ; & a<x<b \\
\frac{R}{\sqrt{\kappa_{n}(x)}} e^{-\int_{b}^{x} \kappa_{n}(y) d y} ; & x \gg b
\end{array}\right.
$$

with

$$
\begin{aligned}
k_{n}(x) & =\sqrt{2\left[E_{n}^{(0)}-V_{t r}(x)\right]}, \\
\kappa_{n}(x) & =i k(x),
\end{aligned}
$$

the wave vectors. $R$ is a constant determined by normalizing the ground-state wave function at $n=0$, that is $\int_{-\infty}^{+\infty}\left|\phi_{0}(x)\right|^{2}=1$ so that 
TABLE III: As in Table $\square$ but for the QT+NGP in Fig. [3(A) with the corrections to the energies obtained via $\phi_{n}(x)$ given by Eq.(41) with the wave vectors (42). $\left\langle E_{G P}\right\rangle_{t}$ and $E_{n}$ are in units of $\hbar \bar{\omega}$, and $\mathcal{G}_{1 d d}$ in $a_{h o}$.

\begin{tabular}{|c|c|c|c|c|c|}
\hline $\mathcal{G}_{1 d d}$ & $\left\langle E_{G P}\right\rangle_{t}$ & $E_{n}$ & $n$ & $M$ & $\left|\left\langle E_{G P}\right\rangle_{t}-E_{n}\right|$ \\
\hline 173.701 & 23.9272 & 24.8019 & 10 & 60 & 0.87470 \\
245.888 & 31.7848 & 31.409 & 12 & 60 & 0.37580 \\
\hline
\end{tabular}

\section{E. Transition Probabilities}

$$
\begin{aligned}
& \int_{-\infty}^{a} d x\left|\frac{R}{\sqrt{\kappa_{0}(x)}} e^{-\int_{x}^{a} \kappa_{0}(y) d y}\right|^{2}+ \\
& \int_{a}^{b} d x\left|\frac{2 R}{\sqrt{k_{0}(x)}} \sin \left[\int_{x}^{b} k_{0}(y) d y+\frac{\pi}{4}\right]\right|^{2}+ \\
& \int_{b}^{+\infty} d x\left|\frac{R}{\sqrt{\kappa_{0}(x)}} e^{-\int_{x}^{a} \kappa_{0}(y) d y}\right|^{2}=1 .
\end{aligned}
$$

The first-order correction to the unperturbed energy $E_{n}^{(0)}$ [Eq.(39)] via the NGP perturbation becomes

$E_{n}^{(1)}=4 R^{2} A \int_{a}^{b} d x \frac{1}{k_{n}(x)} \sin ^{2}\left[\int_{x}^{b} k_{n}(x) d x+\frac{\pi}{4}\right] e^{-\beta x^{2}}$.

It should be noted, that the wave functions in the regimes outside the QT have not been considered in evaluating $E_{n}^{(1)}$ since they do not overlap with the NGP and therefore do not make any contribution. Along the same lines, the second-order correction yields

$$
\begin{aligned}
& E_{n}^{(2)}=\sum_{\substack{m \neq n \\
m \neq n}} \frac{1}{E_{m}^{(0)}-E_{n}^{(0)}} \times \\
& 4 R^{2} A \int_{a}^{b} d x \frac{1}{\sqrt{k_{n}(x) k_{m}(x)}} e^{-\beta x^{2} \times} \\
& \left.\sin \left[\int_{x}^{b} k_{n}(y) d y+\frac{\pi}{4}\right] \sin \left[\int_{x}^{b} k_{m}(y) d y+\frac{\pi}{4}\right]\right|^{2},
\end{aligned}
$$

where $E_{n}^{(0)}$ is given by (39).

Table III shows again that $\left\langle E_{G P}\right\rangle_{t}$ at the PRs in the QT can be closely matched to the energies of secondorder perturbation theory. It should be emphasized that well-behaved, exact analytic solutions to the Schrödinger equation with a quartic oscillator are hitherto unknown. Although, the Heun function [100] may provide a solution within a restricted range in the neighborhood of the trap center, outside the latter it diverges to very large values. We have found that even a numerical solution by Mathematica yields divergent solutions away from the center (not shown here).

\section{Harmonic Trap}

In what follows, the transition probabilities at which PRs occur in an $\mathrm{HO}+\mathrm{NGP}$ trap are evaluated. Basing on the assumption that the NGP and DDI are perturbations inducing transitions between the different states in the traps, the probabilities for these can be computed according to time-dependent perturbation theory. In this regard, the probability for a transfer from some state $m$ to state $n$ is from standard quantum mechanics textbooks given by

$$
P(n, m, t)=\left|c_{n, m}(t)\right|^{2},
$$

where as usual

$$
c_{n, m}(t)=\frac{\lambda}{i \hbar} \int_{0}^{t} d t^{\prime} e^{i\left(E_{m}^{(0)}-E_{n}^{(0)}\right) t^{\prime} / \hbar}\left\langle\phi_{m}(x)\left|V\left(t^{\prime}\right)\right| \phi_{n}(x)\right\rangle
$$

with $V\left(t^{\prime}\right)$ being a time-dependent perturbation. Considering first $\phi_{n}(x)$ to be the $\mathrm{HO}$ states given by (30) and $V\left(t^{\prime}\right)$ the time-dependent NGP (2), then it is easy to show that the probability introduced by the NGP is (setting $\hbar=1$ )

$$
\begin{aligned}
& P^{D T}(n, m, t)=\mid \frac{\lambda}{i} \frac{1}{\sqrt{\pi 2^{n+m} n ! m !}} \times \\
& \left\{\int_{-\infty}^{+\infty} H_{m}(x) H_{n}(x) e^{-(\beta+1) x^{2}} d x\right\} \times \\
& {\left[A F_{0}(m, n, t)+\left.\delta A\left(F_{+\Omega}(m, n, t)+F_{-\Omega}(m, n, t)\right]\right|^{2},\right.}
\end{aligned}
$$

where

$$
\begin{aligned}
F_{0}(m, n, t) & =\frac{e^{i(m-n) t}-1}{i(m-n)}, \\
F_{+\Omega}(m, n, t) & =\frac{e^{i(m-n+\Omega) t}-1}{2 i(m-n+\Omega)}, \\
F_{-\Omega}(m, n, t) & =\frac{e^{i(m-n-\Omega) t}-1}{2 i(m-n-\Omega)} .
\end{aligned}
$$

On the other hand, to compute the transition probability because of the DDI, the Fourier transform (FT) of the 1D 
dipolar potential $V_{1 D}\left(x-x^{\prime}\right)$, such as the one presented by Sinha and Santos 101], needs to be applied to avoid the singularity in the DDI. This FT reads for a quasi 1D Bose gas

$$
\tilde{V}_{1 D}(k)=\frac{4 \alpha d^{2}}{\ell^{2}}\left[1-\sigma e^{\sigma} \Gamma(0, \sigma)\right],
$$

were $\Gamma(0, \sigma)$ is the incomplete gamma function, and $\sigma=k^{2} \ell^{2} / 4$. Here $\ell=\sqrt{\hbar /(\mu \bar{\omega})}$ is a length scale with $\mu=m / 2$ the reduced mass. The factor $\alpha$ has values between $-1 / 2(\phi=\pi / 2)$ and $1(\phi=0)$ where $\phi$ is the angle between the dipoles and the longitudinal axis of the trap. In the present case $\ell=d_{\rho}$ is the width of the wave function in the transverse direction. Appendix B shows how to cast (50) in trap units, that is $\tilde{V}_{1 D} \rightarrow \tilde{V}_{1 D} /(\hbar \omega)$.

Following Muruganandam [45], the spatial integral required in Eq. (47) for the transition between states $n$ and $m$, namely

$$
\begin{aligned}
& \left\langle\phi_{n}(x)\left|V\left(t^{\prime}\right)\right| \phi_{m}(x)\right\rangle \rightarrow \\
& \frac{N}{2} \int_{-\infty}^{+\infty} d x \int_{-\infty}^{+\infty} d x^{\prime} \phi_{n}^{\star}(x) \phi_{m}\left(x^{\prime}\right) V_{1 D}\left(x-x^{\prime}\right),
\end{aligned}
$$

can be rewritten using the FT [Eq. (50)] with its strength given by (B5) and the FT of the HO functions $\widetilde{\phi}_{n}(k)$ [7] where

$$
\widetilde{\phi}_{n}(k)=\mathrm{FT}\left[\phi_{n}(x)\right]=\frac{1}{\sqrt{\sqrt{\pi} 2^{n} n !}}(-i)^{n} H_{n}(k) e^{-k^{2} / 2} .
$$

This then yields that

$$
\begin{aligned}
& \left\langle\phi_{n}(x)\left|V\left(t^{\prime}\right)\right| \phi_{m}(x)\right\rangle \rightarrow \\
& \frac{N}{2} \frac{1}{2 \pi} \int_{-\infty}^{+\infty} d k \widetilde{\phi}_{n}^{\star}(k) \widetilde{\phi}_{m}(-k) \tilde{V}_{1 D}(k) .
\end{aligned}
$$

As such, the probability for the DDI reads then

$$
\begin{aligned}
& P^{D D I}(n, m, t)=\lambda^{2} \mid \frac{\mathcal{G}_{1 d d}(-i)^{n+m}}{\sqrt{\pi 2^{n+m} n ! m !}} \times \\
& \int_{-\infty}^{+\infty} H_{n}(k) H_{m}(-k) e^{-k^{2}} \times \\
& {\left.\left[1-\frac{k^{2} d_{\rho}^{2}}{4} e^{k^{2} d_{\rho}^{2} / 4} \Gamma\left(0, k^{2} d_{\rho}^{2} / 4\right)\right] F_{0}(m, n, t)\right|^{2} .}
\end{aligned}
$$

The above two probabilities $P^{D T}(n, m, t)[$ Eq. (48)] and $P^{D D I}(n, m, t)$ can be easily evaluated numerically by Mathematica for the integer values of $m$ and $n$. These
TABLE IV: Most probable transitions to the states $n$ at which the GP energies are matched in Table \ for the HO+NGP trap. The transition probability is computed by Eqs. (48), (54), and (55). $\mathcal{G}_{1 d d}$ is in units of $a_{h o}$.

\begin{tabular}{|c|c|c|c|}
\hline $\mathcal{G}_{1 d d}$ & $m$ & $n$ & $\mathcal{P}(n, m, t) / \lambda^{4}$ \\
\hline 29.9297 & 2 & 4 & 0.0261 \\
67.8894 & 5 & 7 & 0.0511 \\
120.4490 & 8 & 10 & 0.1216 \\
191.9880 & 11 & 13 & 0.2210 \\
292.7270 & 16 & 18 & 0.3430 \\
\hline \hline
\end{tabular}

two probabilities are dependent on each other and therefore the total probability is

$$
\mathcal{P}(n, m, t)=P^{D T}(n, m, t) P^{D D I}(n, m, t) .
$$

Table IV displays $P(n, m, t)$ (in units of arbitrary $\left.\lambda^{4}\right)$ for the most probable transitions to the states $n$ matched in Table \ for a length of one driving cycle $t=0.1$. In this, it is assumed that $\lambda \ll 1$. It should be noted, that a significant number of $m \leftrightarrow n$ transitions turned out to be prohibited with identically zero values for $P(n, m, t)$. This explains the absence of PRs for values of $\mathcal{G}_{1 d d}$ with a time-averaged GP that nevertheless can be matched to one of the energy levels given by perturbation theory in Sec. IVB Thus there are two conditions for PRs to occur: (1) its energy should match one of the trap levels and (2) the transition to this level should have a high probability.

\section{Box Potential}

As in the previous section, the goal is to find the most probable transitions at which the PRs arise in the $\mathrm{BOX}+\mathrm{NGP}$ trap. In this case,

$$
\begin{aligned}
& P^{D T}(n, m, t)=\mid \frac{\lambda}{i} \frac{1}{L_{x}} \times \\
& \left\{\int_{-L_{x}}^{+L_{x}} \cos \left(\frac{m \pi x}{2 L_{x}}\right) \cos \left(\frac{n \pi x}{2 L_{x}}\right) e^{-\beta x^{2}} d x\right\} \times \\
& {\left.\left[A F_{0}(m, n, t)+\delta A\left(F_{+\Omega}(m, n, t)+F_{-\Omega}(m, n, t)\right)\right]\right|^{2}}
\end{aligned}
$$

and 
TABLE V: As in Table IV but for the BOX+NGP trap. $\mathcal{G}_{1 d d}$ is in units of $a_{h o}$.

\begin{tabular}{|c|c|c|c|}
\hline $\mathcal{G}_{1 d d}$ & $m$ & $n$ & $\mathcal{P}(n, m, t) / \lambda^{4}$ \\
\hline 7.0 & 129 & 131 & 0.0048 \\
78.0 & 101 & 109 & 0.2623 \\
146.882 & 61 & 89 & 1.1894 \\
216.331 & 65 & 87 & 2.8498 \\
285.841 & 81 & 85 & 3.5049 \\
356.060 & 71 & 73 & 71.203 \\
\hline \hline
\end{tabular}

$$
\begin{aligned}
& P^{D D I}(n, m, t)=\mid \frac{\lambda}{i} 4 L_{x}^{2} \mathcal{G}_{1 d d} \times \\
& \int_{-K_{0}}^{+K_{0}}\left[\frac{\sin \left(k L_{x}-\frac{n \pi}{2}\right)}{2 k L_{x}-n \pi}+\frac{\sin \left(k L_{x}+\frac{n \pi}{2}\right)}{2 k L_{x}+n \pi}\right] \times \\
& {\left[\frac{\sin \left(k L_{x}-\frac{m \pi}{2}\right)}{2 k L_{x}-m \pi}+\frac{\sin \left(k L_{x}+\frac{m \pi}{2}\right)}{2 k L_{x}+m \pi}\right] \times} \\
& {\left.\left[1-\frac{k^{2} d_{\rho}^{2}}{4} e^{k^{2} d_{\rho}^{2} / 4} \Gamma\left[0, k^{2} d_{\rho}^{2} / 4\right]\right] F_{0}(m, n, t)\right|^{2},}
\end{aligned}
$$

where the FT of $\cos \left(n \pi x /\left(2 L_{x}\right)\right)$ inside the box is

$$
\begin{aligned}
& F T\left[\cos \left(n \pi x /\left(2 L_{x}\right)\right)\right]= \\
& 2 L_{x}\left[\frac{\sin \left(k L_{x}-\frac{n \pi}{2}\right)}{2 k L_{x}-n \pi}+\frac{\sin \left(k L_{x}+\frac{n \pi}{2}\right)}{2 k L_{x}+n \pi}\right] .
\end{aligned}
$$

In (57), $K_{0}$ is a cutoff momentum introduced to simplify the integration, but which would still give the same result as going to infinite values. Table $\nabla$ is as in Table IV but for the box. The most probable transitions are found to come from relatively high states $m$ towards $n$ matched in Table [I]

Finally, it must be noted that because of the divergent wavefunction of the QT +NGP already mentioned in Sec. IVE , an analytical evaluation of the $P^{D T}$ and $P^{D D I}$ is currently extremely difficult if not impossible, and is therefore left for the future.

\section{SUMMARY AND CONCLUSIONS}

In summary then, we have reported mean-field parametric resonances (PRs) in a one-dimensional (1D) dipolar Bose-Einstein condensate (DBEC) excited by a negative Gaussian potential (NGP) with a periodically oscillating depth. The PRs have been detected by the signal energy [44, a quantity that closely resembles the timeaverage of the square of the energy (in this work the kinetic energy). It is similar to the root-mean-squared (RMS) value of an oscillating electrical signal arising from an alternating voltage or current source. The latter NGP was for modelling a red laser light source with oscillating intensity in a manner similar to Refs. 1, 43].

By a matching of the PR energies to energy levels computed by time-independent perturbation theory we were able to characterize the energy-level structure (ELS) of a DBEC in a complex trap, i.e., a trap to which an NGP has been added. Within the purpose of the latter characterization, a few different traps have been applied to test the effect of confining geometry and its interplay with dipole-dipole interactions (DDI) on the PRs. It turns out the DDI play an important role in defining the amplitude of the PRs and their energies. The DDI reduces the depth of the effectve mean-field potential, thereby controlling the occupancy of the NGP, and in turn the positions and strengths of the PRs.

The key feature of these PRs is that their properties are sensitive to the ELS of the confining potential, i.e., external trap+NGP. The trapping geometry determines the wavefunction that describes the system, and in turn the wavefunction in conjunction with the NGP and DDI determine the transition probabilities between different quantum states of the confinement. The PRs correspond to the most probable transitions determined from timedependent perturbation theory. Nevertheless, it is rather surprising that these PRs correspond only to specific values of the principal quantum numbers of high transitional probabilities, say $m$ and $n$, and that they do not arise at other values $(m, n)$ of comparable, or slightly lower, probabilities. One might even begin to assume the presence of magic numbers for $n$ and $m$, although this assertion needs to be proven.

Most importantly, DBEC PRs could be astonishingly produced by the Lagrangian variational method (LVM) [46] that approximates the wavefunctions by a simple Gaussian Ansatz. However, it was not possible to use LVM for the box and quartic trap because the Gaussian Ansatz had been originally designed for the harmonic trapping case. The LVM PRs are a further manifestation of the fact that these resonances are an inherent feature present in the DBECs awakened by external driving agents.

The present work can also be somewhat related to a previous study on resonances and dynamical fragmentation in a stirred BEC 102 in which a series of PRs in the total energy arises as the rotational frequency is increased. In this regard, the authors conclude that fragmentation of the gas appears simultaneously with the resonant absorption of energy and angular momentum from the external excitation agent. Whereas their PRs are associated with fragmentation of the BEC, in the present mean-field GP formulation there is no fragmentation. The PRs of the current work arise because of resonant absorption of momentum from the dynamic NGP. This momentum arises from the time-varying force Eq.(3) which is the gradient of the NGP that acts along the 
length of the BEC.

The previous results in Secs. III $A-C$ are also a manifestation for the role of a mean-field dipolar nonlinearity in defining the properties of the PRs and -via its interplay with the trapping geometry- also their spacings $\Delta \mathcal{G}_{1 d d}$. One question that arises is if similar results could be obtained by simulations of the same systems using the many-body Schrödinger equation with DDI. In this case, the DDI do not arise in the form of a nonlinearity and it would be interesting to make comparisons between the many-body effects of the DDI and that of the DDI nonlinearity.

\section{Acknowledgement}

The calculations were performed on the PARADOXIV supercomputing facility at the Scientic Computing Laboratory of the Institute of Physics Belgrade, supported in part by the Ministry of Education, Science and Technological Development of the Republic of Serbia under project No. ON171017. We thank William J Mullin (UMASS, Amherst USA) for insightful comments on an earlier version of this manuscript that helped us to improve it substantially. The authors declare that there are no conflicts of interest.

\section{Appendix A: Numerics}

The TDGPE [Eq. (77)] is solved numerically via the split-step Crank-Nicolson $(\mathrm{CN})$ method [52, 80] in real time for the $\mathrm{HO}(p=2)$, QT $(p=4)$, and box potential traps. For the HO and QT, it is propagated along a grid of $N_{x}=6000$ pixels of size $\Delta x=0.01$ with $L_{x}=1$ in Eq.(11). The time step chosen is $\Delta t=1 \times 10^{-4}$, the number of time steps in the transient run is set to $N_{\text {pas }}=10^{6}$, and the same in the final run $N_{\text {run }}=10^{6}$. For the box potential $N_{x}=2048, \Delta x=0.05, L_{x}=51.2$, $\Delta t=0.00025, N_{\text {pas }}=200000$, and $N_{\text {run }}=200000$. In all cases, the system is initialized with a stationary NGP of depth $A=-30$ via the imaginary-time CN method for a number of $N_{s t p}=2 \times 10^{5}$ time steps, and then taken through $N_{\text {pas }}=20000$ steps in the transient, and ending with $N_{\text {run }}=20000$ steps in the final run. The time step $\Delta t$ is the same as in the corresponding real-time simulations. In the $\mathrm{CN}$ codes that have been used, the final run (NRUN) just complements the transient one (NPAS). The latter could be the stage during which the BEC is allowed to evolve and relax to a stable state. The final run could then be the stage where the BEC is optionally excited by an external agent in order to examine its ensuing dynamics. The authors of the code originally separated the transient and final runs for organizational purposes.
This is in order to have data files in the relaxation stage of the BEC towards a stable state separate from the one where this stable BEC is suddenly excited by an external driving force. In contrast, the present work considers a BEC that is continuously excited in both transient and final runs, so there is no difference between both of them.

Sets of runs were performed each at a fixed value of $\mathcal{G}_{1 D}$. For each $\mathcal{G}_{1 D}$, the system was scanned along a range of $\mathcal{G}_{1 d d}$ from 0 to 400 in steps of 2 . It should be emphasized that for each $\mathcal{G}_{1 d d}$ there was a run. This large number of runs has been conducted in the form of parallel array jobs on the high performance computational cluster of the Scientific Computing Laboratory of the Institute of Physics in Belgrade, Serbia. Each simulation took about five days to finish. In essence, this has been a heavily computational project.

\section{Appendix B: Units of $V_{1 D}(k)$}

Beginning with the dipole moment $d$ where 52 .

$$
d^{2}=\frac{\mu_{0} \tilde{\mu}^{2}}{4 \pi}
$$

and the dipolar scattering length

$$
a_{d d}=\frac{\mu_{0} \tilde{\mu}^{2} m}{12 \pi \hbar^{2}}
$$

$\tilde{\mu}$ being the magnetic dipole moment and $\mu_{0}$ the magnetic permeability, we substitute (B2) into (B1) to eliminate $\tilde{\mu}$ so that $d^{2}=3 \hbar^{2} a_{d d} / m$. Then the rescaling $d^{2} \rightarrow$ $d^{2} /(\hbar \omega)$ yields

$$
\tilde{d}^{2}=\frac{d^{2}}{\hbar \omega}=\frac{3 \hbar^{2} a_{d d}}{m \hbar \omega}=3 \tilde{a}_{d d} a_{h o}^{3}
$$

where $\tilde{a}_{d d}=a_{d d} / a_{h o}$. Hence, the coefficient of Eq.(50) becomes in trap units

$$
\frac{4 \alpha \tilde{d}^{2}}{d_{\rho}^{2}}=\frac{4 \alpha}{\tilde{d}_{\rho}^{2}} 3 \tilde{a}_{d d} a_{h o}
$$

with $\tilde{d}_{\rho}=d_{\rho} / a_{h o}$. Using Eq.(13) for $\mathcal{G}_{1 d d}$, one eventually gets that the coefficient with $\alpha=-1 / 2$ is

$$
\frac{4 \alpha d^{2}}{\ell^{2}} \rightarrow \frac{4 \pi \mathcal{G}_{1 d d} a_{h o}}{N}
$$

[1] S. Balik, A. L. Win, and M. D. Havey, Phys. Rev. A 80, 023404 (2009), URL
https://link.aps.org/doi/10.1103/PhysRevA.80.023404 
[2] R. R. Sakhel and A. R. Sakhel, J. Phys: Condens. Matter 32, $315401 \quad$ (2020), URL https://iopscience.iop.org/article/10.1088/1361-648X/267606I). Landau and E. Lifshitz, Mechanics, Course of

[3] J. H. V. Nguyen, M. C. Tsatsos, D. Luo, A. U. J. Lode, G. D. Telles, V. S. Bagnato, and R. G. Hulet, Phys. Rev. X 9, 011052 (2019), URL https://link.aps.org/doi/10.1103/PhysRevX.9.011052

[4] T. Chen, K. Shibata, Y. Eto, T. Hirano, and H. Saito, Phys. Rev. A 100, 063610 (2019), URL https://link.aps.org/doi/10.1103/PhysRevA.100.063610

[5] C.-X. Zhu, W. Yi, G.-C. Guo, and Z.-W. Zhou, Phys. Rev. A 99, 023619 (2019), URL https://link.aps.org/doi/10.1103/PhysRevA.99.023619.

[6] Z.-C. Li, Q.-H. Jiang, Z. Lan, W. Zhang, and L. Zhou, Phys. Rev. A 100, 033617 (2019), URL https://link.aps.org/doi/10.1103/PhysRevA.100.033617.

[7] A. R. Sakhel and R. R. Sakhel, Journal of Low Temperature Physics 190, 120 (2018), ISSN 1573-7357, URL https://doi.org/10.1007/s10909-017-1826-7

[8] P. Molignini, L. Papariello, A. U. J. Lode, and R. Chitra, Phys. Rev. A 98, 053620 (2018), URL https://link.aps.org/doi/10.1103/PhysRevA.98.053620.

[9] S. Robertson, F. Michel, and R. Parentani, Phys. Rev. D 98, 056003 (2018), URL https://link.aps.org/doi/10.1103/PhysRevD.98.056003.

[10] S. Lellouch, M. Bukov, E. Demler, and N. Goldman, Phys. Rev. X 7, 021015 (2017).

[11] I. Vidanović, H. Al-Jibbouri, A. Balaž, and A. Pelster, Physica Scripta T149, 014003 (2012), URL https://doi org/10.1088\%2F0031-8949\%2F 2012\%2Ft 149\%2F03134

[12] A. Posazhennikova, M. Trujillo-Martinez, and J. Kroha, Phys. Rev. Lett. 116, 225304 (2016), URL https://link.aps.org/doi/10.1103/PhysRevLett.116.225\$304

[13] D. Kobyakov, V. Bychkov, E. Lundh, A. Bezett, and M. Marklund, Phys. Rev. A 86, 023614 (2012), URL https://link.aps.org/doi/10.1103/PhysRevA.86.023614

[14] J.-K. Xue, G.-Q. Li, A.-X. Zhang, and P. Peng, Phys. Rev. E 77, 016606 (2008), URL https://link.aps.org/doi/10.1103/PhysRevE.77.016606. [36]

[15] P. Engels, C. Atherton, and M. A. Hoefer, Phys. Rev. Lett. 98, 095301 (2007), URL https://link.aps.org/doi/10.1103/PhysRevLett.98.0953037

[16] A. I. Nicolin, R. Carretero-González, and P. G. Kevrekidis, Phys. Rev. A 76, 063609 (2007), URL https://link.aps.org/doi/10.1103/PhysRevA.76.063609

[17] M. Krämer, C. Tozzo, and F. Dalfovo, Phys. Rev. A 71, $061602 \quad$ (2005), URL https://link.aps.org/doi/10.1103/PhysRevA.71.061602

[18] C. Tozzo, M. Krämer, and F. Dalfovo, Phys. Rev. A 72, 023613 (2005), URL https://link.aps.org/doi/10.1103/PhysRevA.72.023613.

[19] T. Chen and B. Yan, Phys. Rev. A 98, $063615 \quad$ (2018), URL https://link.aps.org/doi/10.1103/PhysRevA.98.063615

[20] Sabari, Subramaniyan and Kumar, R. Kishor, Eur. Phys. J. D 72, 48 (2018), URL https://doi.org/10.1140/epjd/e2018-80354-2.

[21] Cairncross, William and Pelster, Axel, Eur. Phys. J. D 68, $106 \quad$ (2014), URL https://doi.org/10.1140/epjd/e2014-40835-x

[22] I. Vidanović, A. Balaž, H. Al-Jibbouri, and A. Pelster, Phys. Rev. A 84, 013618 (2011), URL https://link.aps.org/doi/10.1103/PhysRevA.84.013618

[23] V. I. Arnold, A. Weinstein, and K. Vogtmann, Mathe- matical Methods of Classical Mechanics, Graduate Texts in Mathematics, vol. 60 (Springer,Berlin, 1989).

Theoretical Physics, vol. 1 (Butterworth-Heinemann, Oxford, 1976).

[25] A. Armaroli and F. Biancalana, Phys. Rev. A 87, $063848 \quad$ (2013), URL https://link.aps.org/doi/10.1103/PhysRevA.87.063848

[26] F. Beato and A. Palacios-Laloy, EPJ Quantum Technology 7, 1 (2020).

[27] N. V. Fomin, O. L. Shalaev, and D. V. Shantsev, Journal of Applied Physics 81, 8091 (1997), https://doi.org/10.1063/1.365417, URL https://doi.org/10.1063/1.365417

[28] M. Calvo, Phys. Rev. B 60, 10953 (1999), URL https://link.aps.org/doi/10.1103/PhysRevB.60.10953

[29] G. Hackenbroich, B. Rosenow, and H. A. Weidenmüller, Phys. Rev. Lett. 81, 5896 (1998), URL https://link.aps.org/doi/10.1103/PhysRevLett.81.5896

[30] F. Matera, A. Mecozzi, M. Romagnoli, and M. Settembre, Opt. Lett. 18, 1499 (1993), URL http://ol.osa.org/abstract.cfm?URI=ol-18-18-1499

[31] G. Bismut, B. Pasquiou, E. Maréchal, P. Pedri, L. Vernac, O. Gorceix, and B. Laburthe-Tolra, Phys. Rev. Lett. 105, 040404 (2010), URL https://link.aps.org/doi/10.1103/PhysRevLett.105.040404

[32] K. Góral and L. Santos, Phys. Rev. A 66, $023613 \quad$ (2002), URL https://link.aps.org/doi/10.1103/PhysRevA.66.023613 34003 Mishra and R. Nath, Phys. Rev. A 94, $033633 \quad$ (2016), URL https://link.aps.org/doi/10.1103/PhysRevA.94.033633 F. Wächtler and L. Santos, Phys. Rev. A 94, $043618 \quad$ (2016), URL https://link.aps.org/doi/10.1103/PhysRevA.94.043618

[35] B. Schulz, S. Sala, and A. Saenz, New Journal of Physics 17, $065002 \quad$ (2015), URL https://doi.org/10.1088\%2F $1367-2630 \% 2$ F $17 \% 2$ F $6 \% 2 F 065002$

[36] A. R. P. Lima and A. Pelster, Phys. Rev. A 84, $041604 \quad$ (2011), URL https://link.aps.org/doi/10.1103/PhysRevA.84.041604 F. Ancilotto and F. Toigo, Phys. Rev. A 89, $023617 \quad$ (2014), URL https://link.aps.org/doi/10.1103/PhysRevA.89.023617 [38] R. M. Wilson and J. L. Bohn, Phys. Rev. A 83, $023623 \quad$ (2011), URL https://link.aps.org/doi/10.1103/PhysRevA.83.023623 [39] G. P. Agrawal, Phys. Rev. Lett. 59, 880 (1987), URL https://link.aps.org/doi/10.1103/PhysRevLett.59.880

[40] F. Abdullaev, S. Darmanyan, A. Kobyakov, 213 (1996), ISSN 0375-9601, URL http://www.sciencedirect.com/science/article/pii/037596019

[41] S. Ambomo, C. M. Ngabireng, P. T. Dinda, A. Labruyère, K. Porsezian, and B. Kalithasan, J. Opt. Soc. Am. B 25, 425 (2008), URL http://josab.osa.org/abstract.cfm?URI=josab-25-3-425

[42] Michael C. Garrett, Adrian Ratnapala, Eikbert D. van Ooijen, Christopher J. Vale, Kristian Weegink, Sebastian K. Schnelle, Otto Vainio, Norman R. Heckenberg, Halina Rubinsztein-Dunlop, and Matthew J. Davis, Phys. Rev. A 83, 013630 (2011), URL https://doi.org/10.1103/PhysRevA.83.013630

[43] L. W. Clark, L.-C. Ha, C.-Y. Xu, and C. Chin, Phys. and F. Lederer, Physics Letters A 220, 
Rev. Lett. 115, 155301 (2015).

[44] A. V. Oppenheim and G. C. Verghese, Signals, Systems, and Inference (Prentice Hall Signal Processing Series, Pearson, 2015), 1st ed.

[45] P. Muruganandam and S. K. Adhikari, Las. Phys. 22, 813 (2012).

[46] Roger Sakhel and Asaad Sakhel, J. Phys. B: At. Mol. Opt. Phys. 50, 105301 (2017).

[47] V. M. Perez-Garcia, H. Michinel, J. I. Cirac, M. Lewenstein, and P. Zöller, Phys. Rev. Lett. 77, 5320 (1996).

[48] V. M. Perez-Garcia, H. Michinel, J. I. Cirac, M. Lewenstein, and P. Zöller, Phys. Rev. A 56, 1424 (1997).

[49] Hamid Al-Jibbouri, Ivana Vidanovic, Antun Balaz, and Axel Pelster, J. Phys. B: At. Mol. Opt. Phys. 46, 065303 (2013).

[50] G. M. Falco, J. Phys. B: At. Mol. Opt. Phys. 42, 215303 (2009).

[51] Qi Wei, Liang Zhao-Xin, and Zhang Zhi-Dong, Chinese Physics Letters 30, 060303 (2013).

[52] R. Kishor Kumar, Luis E. Young-S., Dušsan Vudragović, Antun Balaž, Paulsamy Muruganandam, S.K.Adhikari, Computer Physics Communications 195, 117 (2015), URL

[53] G. D. Bruce, S. L. Bromley, G. Smirne, L. Torralbo-Campo, and D. Cassettari, Phys. Rev. A 84, 053410 (2011), URL https://link.aps.org/doi/10.1103/PhysRevA.84.053410

[54] M. Hammes, D. Rychtarik, H.-C. Nägerl, and R. Grimm, Phys. Rev. A 66, 051401 (2002), URL https://link.aps.org/doi/10.1103/PhysRevA.66.051401

[55] C. Tuchendler, A. M. Lance, A. Browaeys, Y. R. P. Sortais, and P. Grangier, Phys. Rev. A 78, 033425 (2008), URL https://link.aps.org/doi/10.1103/PhysRevA.78.033425

[56] D. M. Stamper-Kurn, H.-J. Miesner, A. P. Chikkatur, S. Inouye, J. Stenger, and W. Ketterle, Phys. Rev. Lett. 81, 2194 (1998), URL https://link.aps.org/doi/10.1103/PhysRevLett.81.2194

[57] D. Comparat, A. Fioretti, G. Stern, E. Dimova, B. L. Tolra, and P. Pillet, Phys. Rev. A 73, 043410 (2006), URL https://link.aps.org/doi/10.1103/PhysRevA.73.043410

[58] D. Jacob, E. Mimoun, L. D. Sarlo, M. Weitz, J. Dalibard, and F. Gerbier, New J. Phys. 13, $065022 \quad$ (2011), URL https://doi.org/10.1088\%2F1367-2630\%2F 13\%2F6\%2F0650225

[59] T. L. Gustavson, A. P. Chikkatur, A. E. Leanhardt, A. Görlitz, S. Gupta, D. E. Pritchard, and W. Ketterle, Phys. Rev. Lett. 88, 020401 (2001), URL https://link.aps.org/doi/10.1103/PhysRevLett.88.020401

[60] M. D. Barrett, J. A. Sauer, and M. S. Chapman, Phys. Rev. Lett. 87, 010404 (2001), URL https://link.aps.org/doi/10.1103/PhysRevLett.87.010404

[61] M. Schulz, H. Crepaz, F. Schmidt-Kaler, J. Eschner, and R. Blatt, J. Mod. Opt. 54, 1619 (2007), https://doi.org/10.1080/09500340600861740, URL https://doi.org/10.1080/09500340600861740.

[62] N. P. Proukakis, J. Schmiedmayer, and H. T. C. Stoof, Phys. Rev. A 73, 053603 (2006), URL https://link.aps.org/doi/10.1103/PhysRevA.73.053603.

[63] R. B. Diener, Biao Wu, M. G. Raizen, and Q. Niu, Phys. Rev. Lett. 89, 070401 (2002), URL https://link.aps.org/doi/10.1103/PhysRevLett.89.070401
[64] T. Aioi, T. Kadokura, T. Kishimoto, and H. Saito, Phys. Rev. X 1, 021003 (2011), URL https://link.aps.org/doi/10.1103/PhysRevX.1.021003

[65] H. Uncu, D. Tarhan, E. Demiralp, and O. E. Müstecaplioglu, Las. Phys. 18, 331 (2008), URL https://doi.org/10.1134/S1054660X08030237

[66] C. Weitenberg, S. Kuhr, K. Mølmer, and J. F. Sherson, Phys. Rev. A 84, 032322 (2011), URL https://link.aps.org/doi/10.1103/PhysRevA.84.032322

[67] A. V. Carpentier, J. Belmonte-Beitia, H. Michinel, and M. I. Rodas-Verde, J. of Mod. Opt. 55, 2819 (2008), https://doi.org/10.1080/09500340802209763, URL https : //doi .org/10.1080/09500340802209763.

[68] N. G. Parker, N. P. Proukakis, M. Leadbeater, and C. S. Adams, Phys. Rev. Lett. 90, 220401 (2003), URL https://link.aps.org/doi/10.1103/PhysRevLett.90.220401

[69] C. J. Pethick and S. H., Bose-Einstein Condensation in Dilute Gases (Cambridge University Press, Cambridge, 2008), 2nd ed.

[70] T. Lahaye, C. Menotti, L. Santos, M. Lewenstein, and T. Pfau, Reports on Progress in Physics 72, 126401 (2009), URL https://doi.org/10.1088\%2F0034-4885\%2F72\%2F12\%2F 126401 10455515001344(O'Dell, S. Giovanazzi, and C. Eberlein, Phys. Rev. Lett. 92, 250401 (2004), URL https://link.aps.org/doi/10.1103/PhysRevLett.92.250401

[72] S. Yi and L. You, Phys. Rev. A 61, 041604 (2000), URL https://link.aps.org/doi/10.1103/PhysRevA.61.041604

[73] J. L. Bohn, M. Cavagnero, and C. Ticknor, New Journal of Physics 11, 055039 (2009), URL https://doi.org/10.1088\%2F1367-2630\%2F11\%2F5\%2F055039

[74] K. Góral, K. Rzażewski, and T. Pfau, Phys. Rev. A 61, 051601 (2000), URL https://link.aps.org/doi/10.1103/PhysRevA.61.051601

[75] A. J. Olson, D. L. Whitenack, and Y. P. Chen, Phys. Rev. A 88, 043609 (2013), URL https://link.aps.org/doi/10.1103/PhysRevA.88.043609

76] T. Koch, T. Lahaye, J. Metz, B. Fröhlich, A. Griesmaier, and T. Pfau, Nat. Phys. 4, 218 (2008), URL https://doi.org/10.1038/nphys887

[77] M. Lu, N. Q. Burdick, S. H. Youn, and B. L. Lev, Phys. Rev. Lett. 107, 190401 (2011), URL https://link.aps.org/doi/10.1103/PhysRevLett.107.190401

[78] S. H. Youn, M. Lu, U. Ray, and B. L. Lev, Phys. Rev. A 82, 043425 (2010), URL https://link.aps.org/doi/10.1103/PhysRevA.82.043425 A. Filinov and M. Bonitz, Phys. Rev. A 86, 043628 (2012), URL https://link.aps.org/doi/10.1103/PhysRevA.86.043628

80] P. Muruganandam and S. K. Adhikari, Computer Physics Communications 180, 1888 (2009), URL https://www.sciencedirect.com/science/article/pii/S0010465

[81] D. Vudragović, I. Vidanović, A. Balaž, P. Muruganandam, and S. K. Adhikari, Computer Physics Communications 183, 2021 (2012), URL https://www.sciencedirect.com/science/article/pii/S0010465

[82] V. Lončar, A. Balaž, A. Bogojević, S. Skrbić, P. Muruganandam, and S. K. Adhikari, Computer Physics Communications 200, 406 (2016), URL https://www.sciencedirect.com/science/article/pii/S0010465 [83] V. Lončar, L. E. Young-S., S. Skrbić, P. Muruganandam, S. K. Adhikari, and A. Balaž, Computer Physics Communications 209, 190 (2016), ISSN 0010-4655, URL 
http://www.sciencedirect.com/science/article/pii/S00104655t630Z2Zd2:i.org/10.1007/s10909-018-2068-z.

[84] L. Young-S., D. Vudragović, P. Muruganan- [93] R. Onofrio, C. Raman, J. M. Vogels, J. R. Abo-Shaeer, dam, S. K. Adhikari, and A. Balaž, Computer Physics Communications 204, 209 (2016), URL A. P. Chikkatur, and W. Ketterle, Phys. Rev. Lett. 85, 2228 (2000). https://www.sciencedirect.com/science/article/pii/S019046\$5R3A0733ws, M.-O. Mewes, N. J. van Druten, D. S.

[85] B. Satarić, V. Slavnić, A. Belic, A. Balaž, P. Muruganandam, and S. K. Adhikari, Computer Physics Communications 200, 411 (2016), URL Durfee, D. M. Kurn, and W. Ketterle, Science 273, 84 (1996).

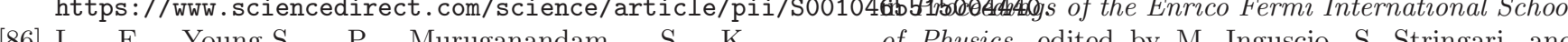
Adhikari, V. Lončar, D. Vudragović, and A. Balaž, Computer Physics Communications 220, 503 (2017), ISSN 0010-4655, URL

[87] R. Kishor Kumar, V. Lončar, P. Muruganandam, S. K. Adhikari, and A. Balaz, Computer Physics Communications 240, 74 (2019), ISSN 0010-4655, URL

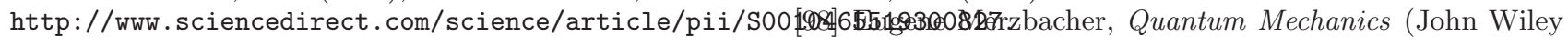

[88] E. E. Nikitin and L. P. Pitaevskii, arxiv:condmat/050868v41 (2005).

[89] Roger R. Sakhel, Asaad R. Sakhel, Humam B. Ghassib, J. Low. Temp. Phys. 173, 177 (2013).

[90] R. R. Sakhel, A. R. Sakhel, H. B. Ghassib, and A. Balaž, The European Physical Journal D 70, 66 (2016), ISSN 1434-6079, URL https://doi.org/10.1140/epjd/e2016-60085-2

[91] A. R. Sakhel, Physica B: Condensed Matter 493, 72 (2016), ISSN 0921-4526, URL of Physics, edited by M. Inguscio, S. Stringari, and C. E. Wieman (IOS Press, Amsterdam, 1999), vol. CXL, p. 67.

[96] J. T. Mendonça, H. Terças, and A. Gam-

[92] R. R. Sakhel and A. R. Sakhel, Journal of Low Temperature Physics 194, 106 (2019), ISSN 1573-7357, URL

[102] M. C. Tsatsos and A. U. J. Lode, Journal of Low Tem-

[99] D. J. Griffiths and D. F. Schröter, Introduction to Quantum Mechanics (Cambridge University Press, 2018), 3rd ed.

[100] A. Ronveaux, ed., Heun's Differential Equations (Oxford, New York, 1995), First ed.

[101] S. Sinha and L. Santos, Phys. Rev. Lett. 99, $140406 \quad$ (2007), URL https://link.aps.org/doi/10.1103/PhysRevLett.99.140406 https://doi.org/10.1007/s10909-015-1335-5. 